\title{
Spectral signature of magnetic flux tubes in sunspot penumbrae
}

\author{
D. A. N. Müller, R. Schlichenmaier, O. Steiner, and M. Stix
}

\author{
Kiepenheuer-Institut für Sonnenphysik, Schöneckstr. 6, 79104 Freiburg, Germany \\ e-mail: schliche, steiner, stix@kis.uni-freiburg.de
}

Received 21 May 2002 / Accepted 2 July 2002

\begin{abstract}
We study the polarization of spectral lines in the penumbra by integrating the radiative transfer equation of polarized light for a three-dimensional model atmosphere of a sunspot. In this model, the Evershed flow is confined to magnetic flux tubes which are embedded in a static background atmosphere, in accordance with the moving tube model of Schlichenmaier et al. (1998a,b). The gradients and/or discontinuities in the fluid velocity and the magnetic field at the flux tube boundaries give rise to asymmetric Stokes profiles. We concentrate on the Stokes- $V$ profiles and study the net circular polarization (NCP) of two photospheric spectral lines of neutral iron, Fe I $630.25 \mathrm{~nm}$ and Fe I $1564.8 \mathrm{~nm}$. The different behavior of these two lines, which are exemplary for atomic spectral lines with a large Landé factor and significantly different wavelength, is based on the difference in excitation potential of the corresponding atomic transitions and the fact that the wavelength dependence of the Doppler shift is linear, while that of the Zeeman splitting is quadratic. We find that the azimuthal variation of the NCP, $\mathcal{N}(\psi)$, is a predominantly antisymmetric function of $\psi$ with respect to the line connecting disk center and spot center (line-of-symmetry) for the infrared line of Fe I $1564.8 \mathrm{~nm}$, while the variation is predominantly symmetric for Fe I $630.25 \mathrm{~nm}$. We show that the antisymmetric variation is caused by anomalous dispersion (Faraday pulsation) and the discontinuity in the azimuthal angle of the magnetic field, which is due to the relative inclination between flux tube and background field. We then compute synthetic $\mathrm{NCP}$ maps of a sunspot and compare them with observational results. Finally, the center-to-limb variation of the $\mathrm{NCP}, \mathcal{N}(\theta)$, of these spectral lines is investigated. We show that the location of the zero-crossing point of $\mathcal{N}(\theta)$ on the center side of the line-of-symmetry represents a diagnostic tool to determine the inclination angle of the Evershed flow: A vanishing NCP on the center-side of the line-of-symmetry is an indirect evidence of downflows in the penumbra.
\end{abstract}

Key words. sunspots - Sun: magnetic fields - Sun: photosphere - techniques: polarimetric - techniques: spectroscopic line: profiles

\section{Introduction}

A sunspot consists of a dark central area, the umbra, surrounded by a brighter region, the penumbra. Observations reveal that the penumbra is highly structured, showing various phenomena on small scales, among which is the photospheric Evershed effect (Evershed 1909), a wavelength shift of spectral lines proportional to the wavelength (St. John 1913). These early measurements indicated that the line shift corresponds to a Doppler shift due to a horizontal material outflow within the penumbra. In the 1960's, it was observationally established that the line shift is accompanied by a line asymmetry, which was soon conjectured to be caused by thin flow channels in the photosphere (Maltby 1964; Rimmele 1995; Schlichenmaier \& Schmidt 2000). Not only the penumbral Stokes-I profiles, but also the other components of the Stokes vector $\boldsymbol{I}=(I, Q, U, V)^{T}$ (defined according to Shurcliff 1962) exhibit Doppler shifts and asymmetries (e.g. Kjeldseth Moe 1968; Beckers \& Schröter 1969; Grigorjev \& Katz 1972; Makita 1979). These asymmetries of Stokes profiles are a

Send offprint requests to: D. A. N. Müller, e-mail: dmueller@kis.uni-freiburg.de direct manifestation of the fine structure of sunspot penumbrae and carry information about gradients along the line-of-sight in the velocity, magnetic field strength, and the direction of the magnetic field vector (see, e.g., Sánchez Almeida \& Lites 1992). Especially the observed net circular polarization (NCP) of spectral lines in the penumbra, i.e. the asymmetry of the Stokes- $V$ profiles, gave rise to different models concerning the structuring of magnetic fields and flows in the penumbra.

Solanki \& Montavon (1993) proposed horizontal magnetic flux tubes, which are embedded in an inclined magnetic field and carry most of the Evershed flow ("uncombed magnetic fields"). Using a three-layered atmospheric model with a horizontal flux sheet as the central layer, they calculated the centerto-limb variation of the NCP, and found that it depends mainly on the discontinuity in magnetic field inclination between two layers. Martínez Pillet (2000) carried this idea one step further by using a more detailed model of the atmospheric background stratification. All these models remain one-dimensional and incorporate a magnetic flux sheet in terms of a change of atmospheric parameters within a certain interval of optical depth.

In this article, we use the moving flux tube model of Schlichenmaier et al. (1998a,b) and describe the spectral 
signature of the radiation emanating from this configuration, in particular the NCP and its spatial variation. Using a threedimensional model atmosphere of a sunspot, we are able to calculate the Stokes vector for any position in the penumbra and arbitrary heliocentric angles.

The moving flux tube model offers an explanation for the increased heat flux in the penumbra relative to the umbra, the penumbral intensity fine structure, and the Evershed flow. The model proposes that the penumbra is heated by magnetic flux tubes in which hot plasma flows up from the deep penumbra to the surface where it cools off by radiation. Inside these tubes, the magnetic field vector is aligned with the flow, while for the direction of the background field, an analytical approximation is developed, based on the formula by Beckers \& Schröter (1969). For the central penumbra, this magnetic field configuration resembles the one of Solanki \& Montavon (1993) and Martínez Pillet (2000).

\section{Radiative transfer of polarized light}

The radiative transfer equation for polarized light describes the variation of the Stokes vector $\boldsymbol{I}=(I, Q, U, V)^{T}$ along a geometrical path $s$ :

$$
\frac{\mathrm{d} \boldsymbol{I}}{\mathrm{d} s}=-\boldsymbol{K} \boldsymbol{I}+\boldsymbol{j}
$$

where $\boldsymbol{K}=\left\{K_{k j}\right\}$ is the total absorption matrix,

$\boldsymbol{K}=\kappa_{\mathrm{c}} \mathbb{1 1}+\kappa_{0} \boldsymbol{\eta}$,

and $\boldsymbol{j}$ the total emission vector,

$\boldsymbol{j}=\kappa_{\mathrm{c}} S_{\mathrm{c}} \boldsymbol{e}_{0}+\kappa_{0} S_{l} \boldsymbol{\eta} \boldsymbol{e}_{0}$.

In theses expressions, $\kappa_{\mathrm{c}}$ and $S_{\mathrm{c}}$ denote the opacity and the source function of the unpolarized continuum, $\kappa_{0}$ the opacity at line center, $S_{l}$ the line source function, 1 the $4 \times 4$ unit matrix and $\boldsymbol{e}_{0}=(1,0,0,0)^{T}$ the unit intensity vector. The line absorption matrix $\boldsymbol{\eta}$ has the form

$\boldsymbol{\eta}=\left(\begin{array}{cccc}\eta_{I} & \eta_{Q} & \eta_{U} & \eta_{V} \\ \eta_{Q} & \eta_{I} & \rho_{V} & -\rho_{U} \\ \eta_{U} & -\rho_{V} & \eta_{I} & \rho_{Q} \\ \eta_{V} & \rho_{U} & -\rho_{Q} & \eta_{I}\end{array}\right)$

and can be decomposed into three components:

(1) A diagonal matrix of $\eta_{I}$ terms describing absorption;

(2) a symmetric matrix containing the $\eta_{Q, U, V}$ terms which describe dichroism, i.e. absorption depending on the polarization state of the radiation;

(3) an antisymmetric matrix containing the $\rho$ terms which represent anomalous dispersion, also known as magnetooptical effects.

In particular, the term $\rho_{V}$, acting alone, would induce a continuous transformation between the Stokes parameters $Q$ and $U$ during propagation. In other words, this corresponds to a rotation of the direction of linear polarization (Faraday rotation). The term $\rho_{Q}$, acting alone, would induce a continuous oscillation between the Stokes parameters $U$ and $V$, which is usually referred to as Faraday pulsation (see, e.g., Landi Degl'Innocenti 2002).

The light path, $s$, is commonly parametrized in terms of the optical depth, $\tau$. For an heliocentric angle $\mu \equiv \cos \theta$ and mass density $\rho$, it is related to the geometrical path by $\mathrm{d} \tau_{\mu} \equiv \kappa \rho \mathrm{d} z / \mu$, where the opacity, $\kappa$, is in general a function of wavelength (here, the continuum opacity at $\lambda=500 \mathrm{~nm}$ is used).

The radiative transfer equation, Eq. (1), can be formally integrated by employing an evolution operator $\boldsymbol{O}\left(s, s^{\prime}\right)$ (Gantmacher 1959):

$\boldsymbol{I}(s)=\int_{s_{0}}^{s} \boldsymbol{O}\left(s, s^{\prime}\right) \boldsymbol{j}\left(s^{\prime}\right) \mathrm{d} s^{\prime}+\boldsymbol{O}\left(s, s_{0}\right) \boldsymbol{I}\left(s_{0}\right)$,

where the operator $\boldsymbol{O}\left(s, s^{\prime}\right)$ obeys the relations

$\frac{\mathrm{d}}{\mathrm{d} s} \boldsymbol{O}\left(s, s^{\prime}\right)=-\boldsymbol{K}(s) \boldsymbol{O}\left(s, s^{\prime}\right) \quad$ and $\quad \boldsymbol{O}(s, s)=11$.

\subsection{Numerical solution}

The radiative transfer equation, Eq. (1), is solved numerically by means of the DELO method (Rees et al. 1989) using the DIAMAG implementation of Grossmann-Doerth (1994). This method assumes a piecewise linear modified source function $S(\log (\tau))$ which in turn yields an analytical expression for the evolution operator $\boldsymbol{O}\left(\tau_{k}, \tau_{k+1}\right)$. The underlying physical processes of line formation and their numerical implementation were summarized by Wittmann (1974) and Landi Degl'Innocenti (1976).

\subsection{Convergence of the DELO method}

A suitable measure to quantify the convergence properties of a numerical scheme is the experimental order of convergence, $\mathcal{E}$ (Kröner 1997):

$\mathcal{E} \equiv \ln \left(\frac{\left\|u-u_{h}\right\|}{\left\|u-u_{2 h}\right\|}\right) / \ln \left(\frac{1}{2}\right)$,

where $u$ is the exact solution, $u_{h}$ and $u_{2 h}$ are the numerical solutions for two intervals of discretization, $h$ and $2 h$, and $\|.$. is a norm, e.g. the $L^{1}$-norm, $\|f(x)\|_{L^{1}}=\int_{\Omega}|f(x)| \mathrm{d} x, x \in \Omega$, or the maximum norm, $L^{\infty},\|f(x)\|_{L^{\infty}}=\max _{x \in \Omega}|f(x)|$.

We calculate the experimental order of convergence of the DELO method using a two-component atmospheric model of a magnetic canopy described by Steiner (2000). This model atmosphere consists of two layers which are separated by a discontinuity in the magnetic field, the magnetopause. The lower layer is field-free and possesses a macroscopic velocity field, while the plasma in the upper layer is at rest and is permeated by a vertical magnetic field. In this situation, the individual radiative transfer equations of Eq. (1) decouple and can be solved analytically for certain atmospheric models. For a configuration with a Milne-Eddington atmosphere in the lower and a constant temperature in the upper part, an exact solution was found by Steiner (2000).

Note that no convergence checks can be carried out on the basis of a pure Milne-Eddington atmosphere as its source function is per definition linear in optical depth, and therefore any 


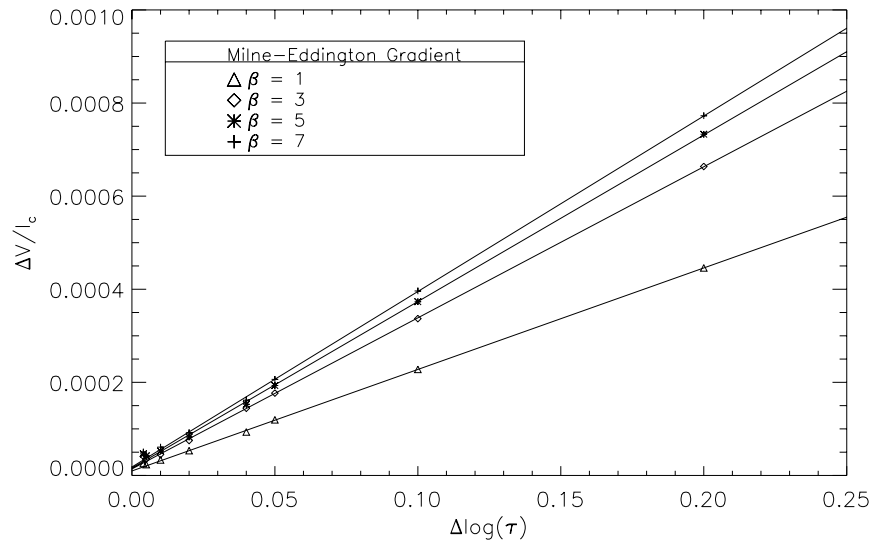

Fig. 1. Maximal error of Stokes- $V$ for an atmospheric model with a magnetopause as a function of discretization $\Delta \log (\tau)$ in optical depth for different gradients $\beta$ of the Milne-Eddington source function.

numerical scheme of first or higher order must yield the exact solution. In the magnetopause configuration which we use here, the source function is continuous, but only piecewise linear, so that reliable tests of the convergence behavior are possible. We obtain for the mean value and the standard deviation of the estimated order of convergence

$\mathcal{E}_{L^{\infty}}=0.96 \pm 0.02$

Figure 1 displays the error of Stokes- $V$ with respect to the maximum norm as a function of the discretization interval $\Delta \log (\tau)$ in optical depth.

\subsubsection{Convergence in the presence of discontinuities}

In the presence of discontinuities in atmospheric parameters (not only magnetic parameters as in the previous section) which occur, e.g., at the boundaries of magnetic flux tubes, numerical schemes do not necessarily converge. As methods of higher order tend to yield oscillatory solutions in the vicinity of discontinuities, a linear method should be employed here. In this respect, the DELO method is suitable for the class of problems we seek to examine in this treatment. In all cases in which no analytical solution of the radiative transfer equation can be found, the order of convergence can be estimated by replacing the exact solution with a reference solution that is assumed to be sufficiently close to the exact one (by using a high number of grid points). Applying the $L^{1}$-norm, we obtain

$\mathcal{E}_{L^{1}}=1.17 \pm 0.03$

and for the maximum norm

$\mathcal{E}_{L^{\infty}}=1.08 \pm 0.04$

where the mean value and its standard deviation are given in each case (details can be found in Müller 2001). This shows that the DELO method is capable of solving the radiative transfer equation even in the presence of discontinuities while maintaining approximately linear convergence behavior.

\section{Asymmetric Stokes- $V$ profiles}

In the simplest case of a magnetohydrostatic atmosphere, the Stokes- $V$ profiles of atomic spectral lines should be antisymmetric (Auer \& Heasley 1978; Landi Degl'Innocenti \& Landi Degl'Innocenti 1981). Observations show, however, that the majority of profiles exhibit in fact asymmetries. A suitable measure for the asymmetry of Stokes- $V$ profiles is the area asymmetry or net circular polarization, $\mathcal{N}$, of a spectral line, which we define as

$\mathcal{N} \equiv \int_{\delta \lambda} V(\lambda) \mathrm{d} \lambda$,

where the interval of integration, $\delta \lambda$, encompasses the whole line profile. The first measurements of the net circular polarization in sunspots were reported by Illing et al. (1974), who measured its center-to-limb variation using a filter of $10 \mathrm{~nm}$ width ("broad-band circular polarization"). They excluded a polarization of the continuum as conjectured by Kemp (1970) as a possible explanation of their findings as the asymmetries were two orders of magnitude higher than the expected values for continuum polarization. Auer \& Heasley (1978) showed that these observations could be explained by assuming macroscopic velocity fields. Furthermore, they proved that velocity gradients are a necessary and sufficient condition to produce a NCP.

\section{Model of the magnetic field in the penumbra}

In this section, we present our model of the magnetic field structure in the penumbra. First, we introduce the coordinate systems which are used, then elaborate on a simple analytical model for the inclination of the background magnetic field, and finally describe the procedure of discretization of a model magnetic flux tube and its embedding in the background model.

\subsection{Coordinate systems}

A position in the surface plane of a sunspot can be given in polar coordinates $(r, \psi)$, with $r$ being the distance from spot center and with $\psi=0^{\circ}$ and $180^{\circ}$ corresponding to the line which connects the disk center with the spot center. This line is also referred to as the line-of-symmetry, since, e.g., the map of the line-of-sight (hereafter LOS) velocity component of a radial outflow is symmetric with respect to this line. As depicted in Fig. 2, we introduce a local cartesian coordinate system $\left(x^{\prime}, y^{\prime}, z^{\prime}\right)$ at $(r, \psi)$. The $z^{\prime}$-axis is parallel to the surface normal $\hat{\boldsymbol{n}}$ and the $x^{\prime}$-axis is parallel to the line-of-symmetry. A vector, $\boldsymbol{B}$, is described by the inclination, $\gamma^{\prime}$, with respect to $\hat{\boldsymbol{n}}$, and the azimuth, $\phi^{\prime}$. The coordinates of $\boldsymbol{B}$ in the local cartesian system are $B_{x^{\prime}}=B \sin \gamma^{\prime} \cos \phi^{\prime}, B_{y^{\prime}}=B \sin \gamma^{\prime} \sin \phi^{\prime}$, and $B_{z^{\prime}}=B \cos \gamma^{\prime}$, with $B \equiv|\boldsymbol{B}|$.

For the calculation of the emanating Stokes vector, the relevant angles of the magnetic and flow field are the angles for the inclination, $\gamma$, and the azimuth, $\phi$, with respect to the LOS. Hence, the local coordinate system has to be rotated around the $y^{\prime}$-axis by the heliocentric angle, $\theta$, which is the angle between the LOS and $\hat{\boldsymbol{n}}$ (see also Title et al. 1993). In the LOS 


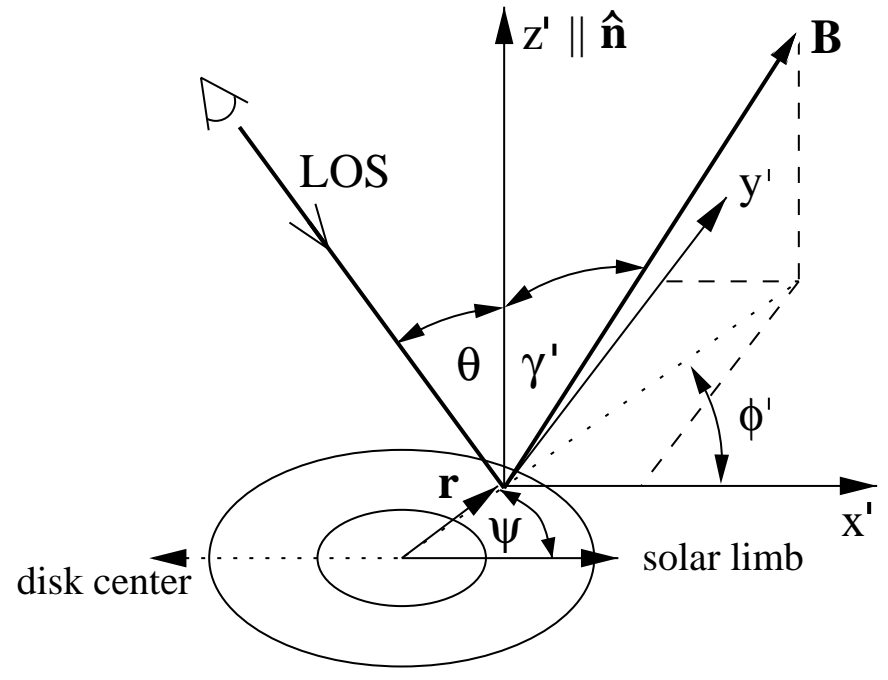

Fig. 2. The magnetic field vector, $\boldsymbol{B}$, at a point in the penumbra with polar coordinates $(r, \psi)$ with respect to the center of the spot, can be described within a local cartesian coordinate system $\left(x^{\prime}, y^{\prime}, z^{\prime}\right)$ with the $\left(x^{\prime}, y^{\prime}\right)$-plane corresponding to the sunspot surface. The $x^{\prime}$-axis is parallel to the line which connects disk and spot center (the line-ofsymmetry), pointing toward the solar limb. The figure sketches the inclination, $\gamma^{\prime}$ with respect to the surface normal, $\hat{\boldsymbol{n}}$, (which is parallel to the $z^{\prime}$-axis), and the azimuth, $\phi^{\prime}$ with respect to the $x^{\prime}$-axis, of $\boldsymbol{B}$. Note that the LOS is within the $\left(x^{\prime}, z^{\prime}\right)$-plane.

coordinate system, the inclination, $\gamma$, and the azimuth, $\phi$, of $\boldsymbol{B}$ are given by

$\gamma=\arccos \left(\cos \gamma^{\prime} \cos \theta-\sin \gamma^{\prime} \cos \phi^{\prime} \sin \theta\right)$,

$\phi=\arctan \left(\frac{\sin \gamma^{\prime} \sin \phi^{\prime}}{\cos \gamma^{\prime} \sin \theta+\sin \gamma^{\prime} \cos \phi^{\prime} \cos \theta}\right)$.

Note that $\phi^{\prime}=0$ implies $\phi=0$. The LOS component of the flow velocity, $v$, with $v_{0}$ being the absolute velocity, is given by

$v=v_{0} \cos \gamma=v_{0}\left(\cos \gamma^{\prime} \cos \theta-\sin \gamma^{\prime} \cos \phi^{\prime} \sin \theta\right)$.

\subsection{Model of the background magnetic field inclination of a sunspot}

From observations, Beckers \& Schröter (1969) deduced the following formula for the magnetic field strength, $B=|\boldsymbol{B}|$, in a sunspot as a function of distance, $r$, from the spot center at a geometrical height $z=z_{0}$ :

$B(r)=B_{0} \frac{1}{1+\left(r / r_{*}\right)^{2}}$.

In this expression, $r_{*}$ denotes the radial distance where the magnetic field strength has decreased by $50 \%$ relative to the field strength at spot center, $B_{0}$. For the inclination at a geometrical height $z=z_{0}$, they found

$\gamma^{\prime}(r)=\frac{\pi}{2} \frac{r}{r_{*}}$

To take into account more recent observations (e.g. Title et al. 1993), according to which the magnetic field of a sunspot is not horizontal at $r=r_{*}$, but shows an inclination of about $5^{\circ}-20^{\circ}$ with respect to the surface, we extend this formula to

$\gamma^{\prime}(r)=\frac{\pi}{2} \frac{r}{r_{\gamma^{\prime}}}$

where $r_{\gamma^{\prime}}$ is the distance from the spot center for which $\gamma^{\prime}=$ $90^{\circ}$. Note that neither Beckers \& Schröter (1969) nor subsequent observers were able to measure a significant azimuthal component. Assuming this component to be zero, we can write $\nabla \cdot \boldsymbol{B}=0$ as

$\frac{1}{r} \frac{\partial}{\partial r}\left(r B_{r}\right)+\frac{\partial B_{z}}{\partial z}=0$.

In this expression, $B_{z}=|B| \cos \gamma^{\prime}$ denotes the vertical and $B_{r}=$ $|B| \sin \gamma^{\prime}$ the horizontal component of the magnetic field. For small height differences $z-z_{0}$, a first order Taylor expansion yields

$B_{z}(r, z) \approx B_{z}\left(r, z_{0}\right)+\left(z-z_{0}\right) \cdot \frac{\partial B_{z}\left(r, z_{0}\right)}{\partial z}$,

$B_{r}(r, z) \approx B_{r}\left(r, z_{0}\right)$.

With Eqs. (15), (17) and (18), one obtains

$$
\begin{aligned}
B_{z}(r, z) \approx & B_{z}\left(r, z_{0}\right)+\left(z-z_{0}\right) \cdot|B| \\
& \cdot\left(-\frac{\sin \gamma^{\prime}}{r}-\frac{\pi}{2 r_{\gamma^{\prime}}} \cos \gamma^{\prime}+\frac{2 r}{r_{*}^{2}} \frac{\sin \gamma^{\prime}}{1+\left(r / r_{*}\right)^{2}}\right) .
\end{aligned}
$$

The inclination $\gamma^{\prime}$ can now be calculated for $z \neq z_{0}$ from $\gamma^{\prime}=\tan ^{-1}\left(B_{r} / B_{z}\right)$, while for $z=z_{0}$ it is given by Eq. (17). In particular, we obtain for the spot center:

$B_{z}(0, z) \approx B_{z}\left(0, z_{0}\right)\left(1-\frac{\pi}{2 r_{\gamma^{\prime}}}\left(z-z_{0}\right)\right)$.

We use this analytical approximation in our penumbral model for the direction of the magnetic field as only the modulus is taken from the model of Jahn \& Schmidt (1994). The denominator $r_{\gamma}^{\prime}$ in Eq. (17) was chosen such that the field lines at the outer penumbral boundary at $z(\tau=1)$ are tangential to the magnetopause of the model of Jahn \& Schmidt (1994). Figure 3 displays the magnetic field in the penumbra in a vertical cut through the flux tube axis. Adopting values of $B_{0}=2700 \mathrm{G}$ and $r_{\gamma^{\prime}}=16800 \mathrm{~km}$ yields a vertical gradient of the magnetic field in the spot center of $\partial B_{z} / \partial z \approx-0.25 \mathrm{G} / \mathrm{km}$, which agrees fairly well with the value of $\partial B_{z} / \partial z \approx-0.3 \mathrm{G} / \mathrm{km}$ as determined from the model of Jahn \& Schmidt (1994) for $r=0$.

\subsection{Magnetic flux tubes in the penumbra}

Our starting point for models of flux tubes in the penumbra is the time-dependent MHD calculation of Schlichenmaier et al. (1998b). We use snapshots of the penumbral flux tube from these simulations as our model flux tubes. The simulation also uses the Jahn \& Schmidt sunspot model with the following parameters: Umbral radius $R_{U}=8000 \mathrm{~km}$, penumbral radius $R_{P}=16000 \mathrm{~km}$, total magnetic flux $\Phi=10^{22} \mathrm{Mx}$. All physical parameters are constant across the flux tube radius, which is computed from flux conservation: $F=\pi r^{2} B=$ const. 


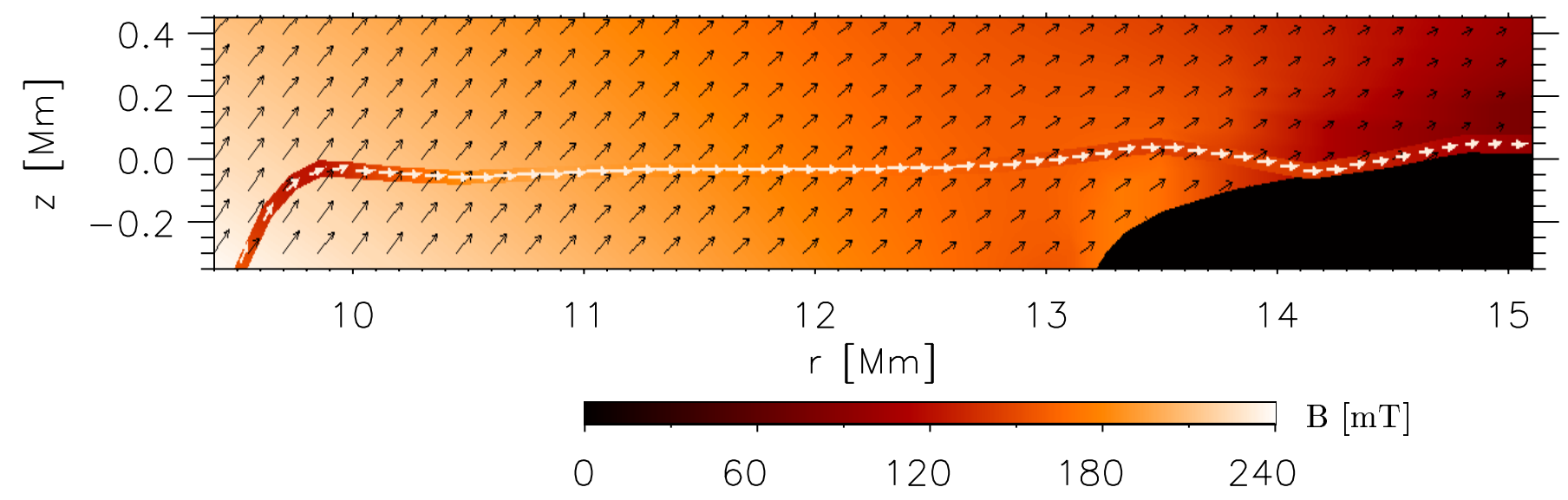

Fig. 3. Amplitude and direction of the magnetic field in the penumbra: vertical cut in the $r$-z-plane. The flow of plasma inside the flux tube is aligned with its magnetic field, while the background is assumed to be static. The gray/color scale indicates the magnetic field strength (according to Jahn \& Schmidt 1994), the black arrows denote the direction of the background magnetic field, according to Eqs. (19) and (20).

\subsubsection{Discretization and embedding of the tube model}

Our goal is to calculate the Stokes vector of the emanating radiation for any position in the penumbra and arbitrary heliocentric angles. For this purpose, we first discretize the axially symmetric flux tube on a regular, i.e. spatially equidistant, three-dimensional grid, using the method of Delaunay triangulation (Delaunay 1934).

When the radiative transfer equation is solved along a LOS which passes through a flux tube, this tube must be spatially resolved, in particular its boundaries, while for computational efficiency it should also be taken into account that the largest part of the LOS passes through the unstructured background atmosphere. There, the thermodynamical parameters depend on height only and the magnetic field is a smooth function of the position $\boldsymbol{x}=(r, z, \psi)$. For this reason, we discretize a three-dimensional box containing the magnetic flux tube, which is subsequently embedded in the parametrized background model. This situation is sketched in Fig. 4.

The LOS is defined by a point $\boldsymbol{x}_{0}=(r, z, \psi)$ in the sunspot and the heliocentric angle, $\theta$, and discretized by a set of points $\mathcal{P}=\left\{\boldsymbol{x}_{i}\right\}_{i=1 \ldots n}$. When the LOS crosses the tube, the physical parameters inside the tube are interpolated onto the corresponding set of points constituting the LOS. A "nearest neighbor" approximation is used to conserve the sharpness of the flux tube boundary. Outside the tube, the thermodynamical parameters and magnetic field strength are interpolated from the background model, while the field inclination is calculated using the analytical approximation given by Eq. (19). The spatial separation of grid points is $h=2-5 \mathrm{~km}$, the length of a box measures between 3000 and 8000 kilometers, the height is $200-800 \mathrm{~km}$, and its width amounts to $100-200 \mathrm{~km}$. An example of the atmospheric stratification along the LOS is shown in Fig. 5.

Our model shares common features with the models of Solanki \& Montavon (1993), Sánchez Almeida et al. (1996), and Martínez Pillet (2000), but in our case, (1) the background is at rest and the magnetic field strength inside the tube is nearly the same as in the background model, and (2) the tube can be viewed from an arbitrary angle.

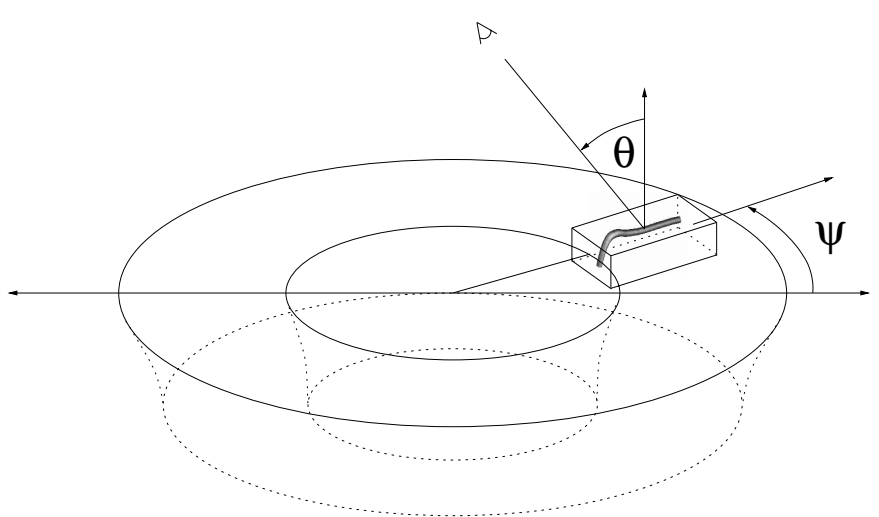

Fig. 4. Flux tube within the discretization box, embedded in the sunspot penumbra.

\section{Spectral signature of penumbral flux tubes}

Asymmetric Stokes- $V$ profiles are formed in the presence of gradients resp. discontinuities in the projections of the velocity field onto the LOS. Gradients or discontinuities in the magnetic field additionally accentuate the asymmetry. The presence of a tube embedded in a penumbral background atmosphere causes discontinuities along a line-of-sight transversing it:

(1) $\Delta v$, the LOS component of the flow velocity (flow channel embedded in a background at rest),

(2) $\Delta \gamma$, the inclination of the magnetic field vector (nearly horizontal flux tube in an inclined background magnetic field), and

(3) $\Delta \phi$, the azimuth of the magnetic field vector with respect to the LOS.

The discontinuity in azimuth, $\Delta \phi$, needs clarification: Although the azimuth of the tube, $\phi_{\mathrm{t}}^{\prime}$, and of the background, $\phi_{\mathrm{b}}^{\prime}$, are the same with respect to the local system, $\Delta \phi=\phi_{\mathrm{t}}-\phi_{\mathrm{b}}$ is non-zero (except for $\theta=0^{\circ}$ or $\psi=0^{\circ}, 180^{\circ}$ ) as a consequence of $\gamma_{\mathrm{t}}^{\prime} \neq \gamma_{\mathrm{b}}^{\prime}$ (cf. Eq. (13)). 

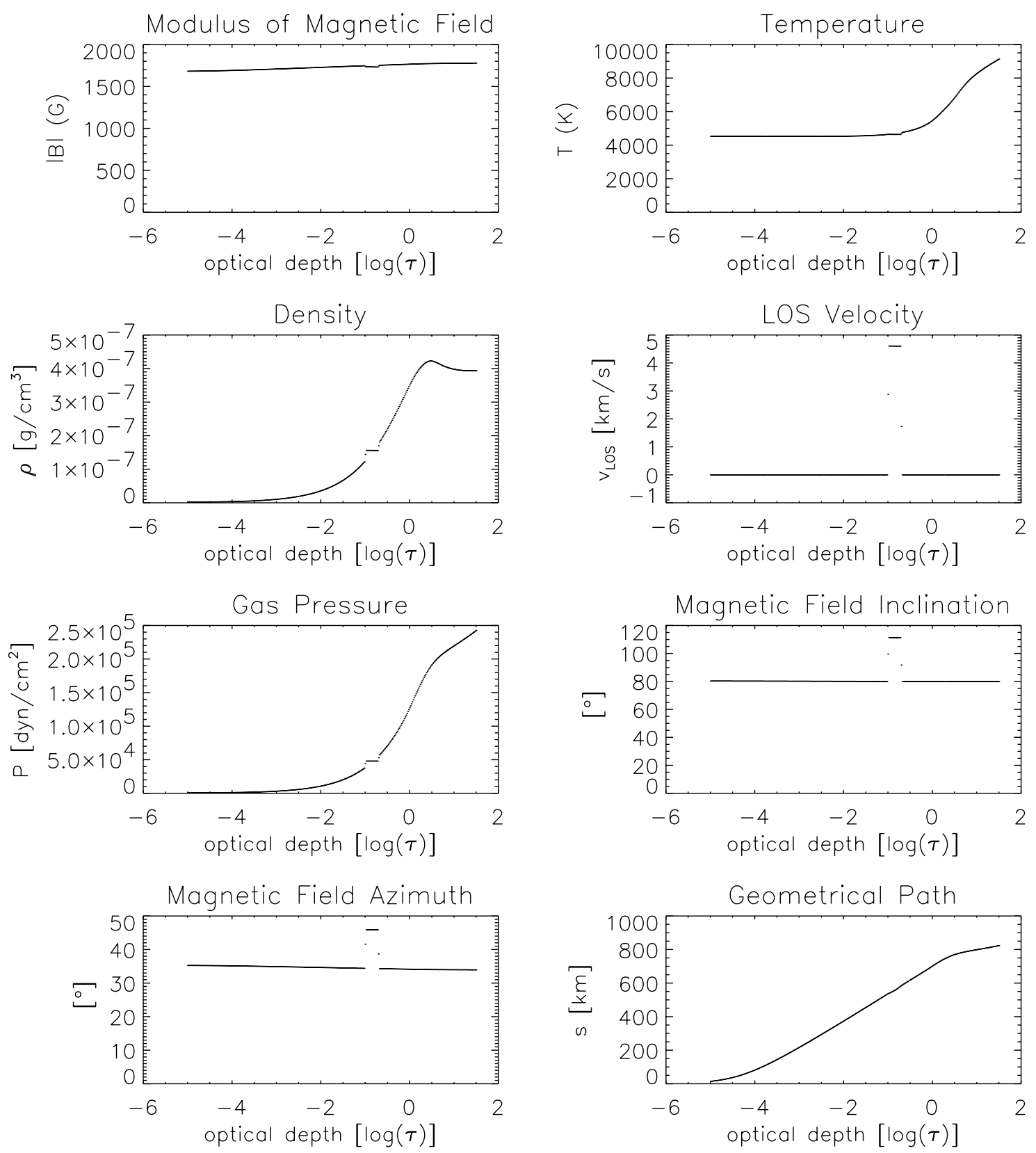

Fig. 5. Physical parameters along the LOS through a magnetic flux tube in the limb-side penumbra (radial position $r=12000 \mathrm{~km}$, heliocentric angle $\theta=30^{\circ}$, azimuthal angle in the sunspot $\psi=220^{\circ}$ ). The plot in the lower right relates the continuum optical depth at $\lambda=500 \mathrm{~nm}$ to the geometrical path along the LOS. A height of $300 \mathrm{~km}$ above $\tau=1$ was chosen as the zero-point of the ordinate, where the optical depth is $\tau=10^{-6}$.

\subsection{Ratio of Zeeman splitting and Doppler shift}

If a tube with a flow speed $v$ is embedded in a static background atmosphere, the absorption profile of a spectral line with rest wavelength $\lambda_{0}$ is Doppler-shifted in this region by $\Delta \lambda_{\mathrm{D}}=\lambda_{0} v / c$, where $v$ is the LOS component of $\boldsymbol{v}$. The spectrum of the light emanating from an atmosphere permeated by flux tubes is determined by the interplay of absorption profiles in the different regions of the atmosphere. The signature of a flux tube is particularly prominent if its flow shifts the maximum of the spectral line contribution of the tube relative to the background contribution. The line strength also plays an important role: If the line absorption coefficient is high, the effect of the flow in the flux tube is swamped by the equivalent width of the spectral line.

Let us now compare the ratio of Doppler shift and Zeeman splitting for a situation in the central penumbra $\left(|\boldsymbol{v}|=12 \mathrm{~km} \mathrm{~s}^{-1}\right.$, $B=1700 \mathrm{G}$ ) for the spectral lines Fe I $630.25 \mathrm{~nm}$ (1) and Fe I $1564.8 \mathrm{~nm}$ (2) which have Landé factors of $g_{1}=2.5$ and $g_{2}=3.0$, respectively. In this example, we assume a horizontal flux tube with the same magnetic field strength as in the background atmosphere. For observations at a heliocentric angle $\theta$, one obtains for the Doppler shift

$\Delta \lambda_{\mathrm{D}, 1}=25 \mathrm{pm} \cdot \sin \theta, \quad \Delta \lambda_{\mathrm{D}, 2}=63 \mathrm{pm} \cdot \sin \theta$, 

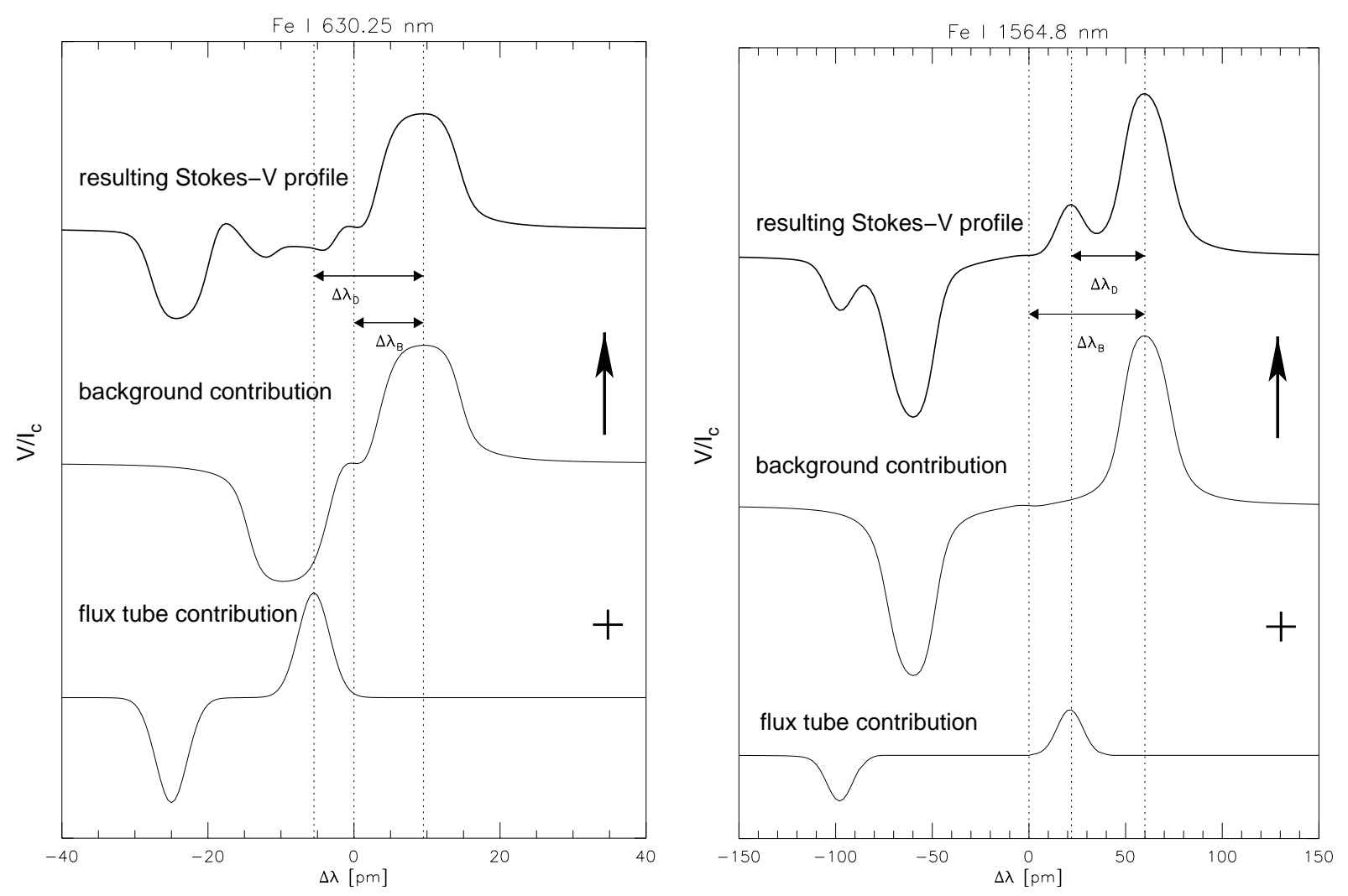

Fig. 6. Sketch of Stokes- $V$ profiles of the lines Fe I $630.25 \mathrm{~nm}$ (top left) and Fe I $1564.8 \mathrm{~nm}$ (top right) for an atmosphere with a magnetic flux tube ( $r=12000 \mathrm{~km}, \theta=35^{\circ}, \psi=180^{\circ}$, i.e. limb-side penumbra). These profiles are composed of a contribution of the background atmosphere and a contribution of the flux tube, which is illustrated by the schematic profiles drawn below.

and for the Zeeman splitting

$\Delta \lambda_{\mathrm{B}, 1}=7.9 \mathrm{pm}, \quad \Delta \lambda_{\mathrm{B}, 2}=58 \mathrm{pm}$.

This yields in the first case a ratio of $\Delta \lambda_{\mathrm{D}, 1} / \Delta \lambda_{\mathrm{B}, 1}=3.16 \cdot \sin \theta$ and in the second case $\Delta \lambda_{\mathrm{D}, 2} / \Delta \lambda_{\mathrm{B}, 2}=1.09 \cdot \sin \theta$. Choosing, e.g., $\theta=35^{\circ}$, we get $\Delta \lambda_{\mathrm{D}, 1} / \Delta \lambda_{\mathrm{B}, 1}=1.81$ and $\Delta \lambda_{\mathrm{D}, 2} / \Delta \lambda_{\mathrm{B}, 2}=$ 0.63. This situation is illustrated in Fig. 6. It is seen that

(i) in the visible wavelength range, the absorption profiles of the flux tube are completely shifted past the absorption profile of the background, while

(ii) for the infrared line, the maxima of the profiles of the flux tube component are located in the wings of the background profiles.

This effect is important to understand the center-to-limb variation of the NCP which will be described in Sect. 8.3.

\section{Center-to-limb variation of Stokes- $V$ profiles}

As a sunspot crosses the solar disk, the projection of the magnetic field and the flow field onto the LOS changes, which offers the possibility to deduce physical properties of flow channels (inclination angle, flow speed), e.g. from the centerto-limb variation (hereafter CLV) of the NCP.

In this section, we present calculations of Stokes- $V$ profiles for Fe I $630.25 \mathrm{~nm}$ and Fe I $1564.8 \mathrm{~nm}$ for a fixed radial position $(r=12000 \mathrm{~km})$, while the center-to-limb-variation of their
NCP will be described in Sect. 8.3. Figure 7 displays these profiles for different heliocentric angles. Additionally, we plot the profiles which result from the atmospheric background model without a flux tube. The profiles were synthesized without consideration of macroturbulence or instrumental broadening and have to be convolved with the respective broadening function for comparison with observations.

For the infrared line (Fig. 7, right panel), the spectral contribution of the flux tube is seen in the form of local maxima in the wings of the background contribution. Their Doppler shift is proportional to the LOS velocity, $v=|\boldsymbol{v}| \cdot \sin \theta$. In the limbside penumbra, the projection of the background magnetic field changes sign at $\theta \approx 30^{\circ}$. The set of points in the penumbra for which the background field is perpendicular to the LOS is referred to as the "magnetic neutral line" (Grigorjev \& Katz 1972) and features strongly asymmetric profiles as those seen in Fig. 7 around $\theta=30^{\circ}$, especially for Fe I $630.25 \mathrm{~nm}$.

In the case of Fe I $630.25 \mathrm{~nm}$ (Fig. 7, left panel), the presence of a flux tube is clearly discernable as well. In this case, however, no cleanly separated spectral contributions (as for the infrared line) are seen, but the profile shapes are partly extremely asymmetric.

To illustrate the different behavior of the two lines, Fig. 8 shows the line-depression contribution functions of Stokes-I (Magain 1986) of the background atmosphere, for the same LOS as in Fig. 5 and at the central wavelength of both spectral lines. The location of the flux tube is indicated by the dotted 
Fe I $630.25 \mathrm{~nm}$

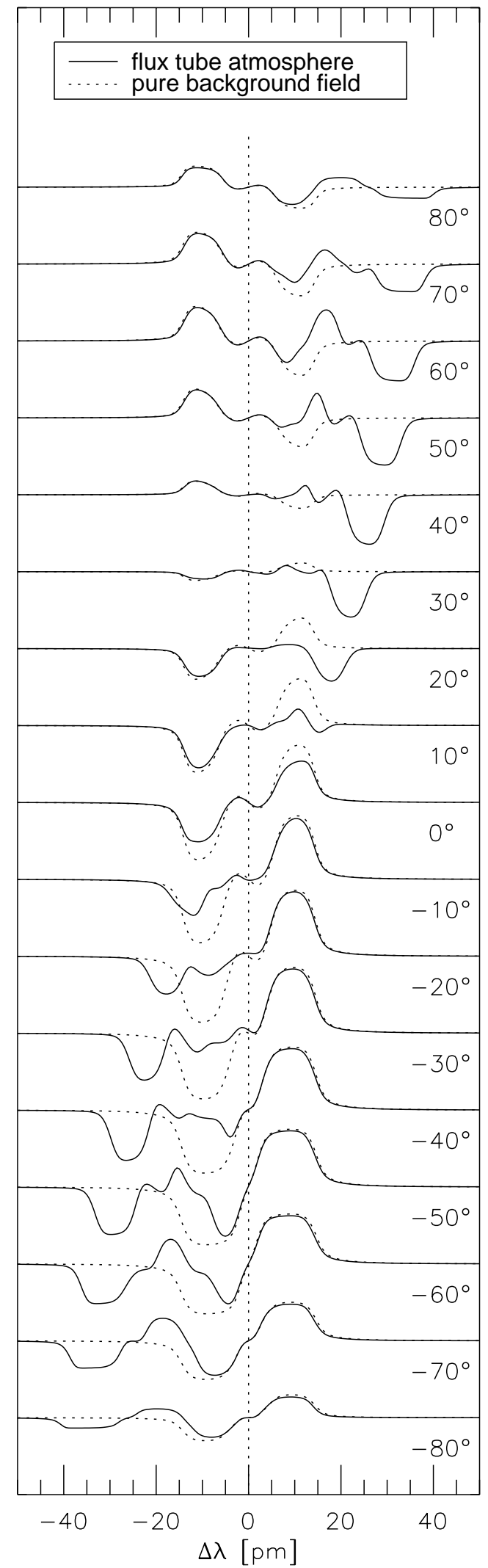

Fe I $1564.8 \mathrm{~nm}$

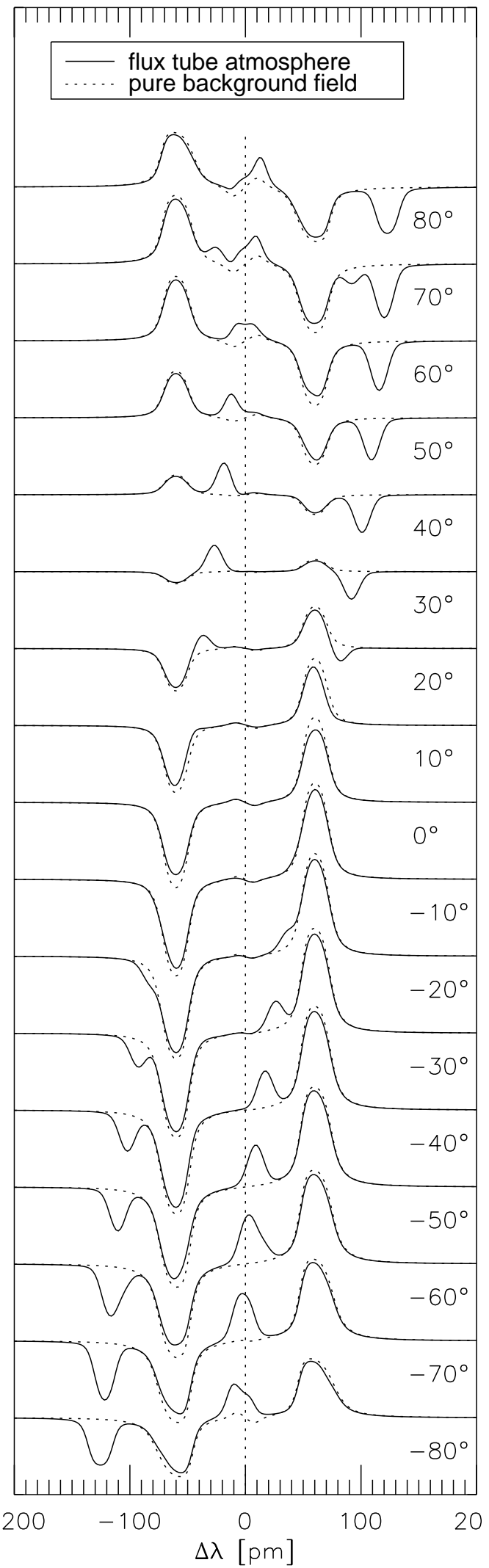

Fig. 7. Center-to-limb variation of Stokes- $V$ in the mid-penumbra $(r=12000 \mathrm{~km})$ for the two lines of Fe I $630.25 \mathrm{~nm}(l e f t)$ and Fe I $1564.8 \mathrm{~nm}$ $($ right $)$. Negative angles denote the center-side penumbra, positive angles the limb side. Solid lines: penumbral model with flux tube, dotted lines: profiles of the background field without flux tube. The vertical distance between two profiles corresponds to $30 \%$ of the continuum intensity in the left panel and $15 \%$ in the right one. Around $\theta=-60^{\circ}$, it is seen that the profiles of Fe I $630.25 \mathrm{~nm}$ saturate. 

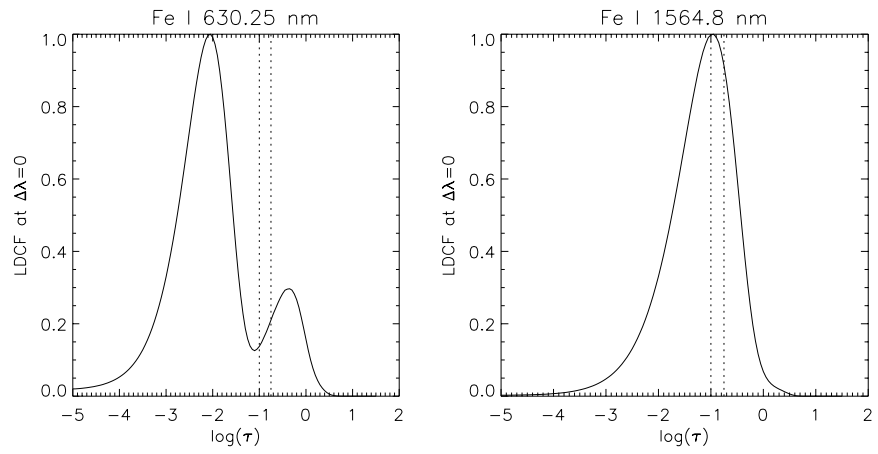

Fig. 8. Line-depression contribution functions of Stokes- $I$ for Fe I $630.25 \mathrm{~nm}$ (left) and Fe I $1564.8 \mathrm{~nm}$ (right) at the central wavelength.

lines. On the one hand, we see that the line of Fe I $630.25 \mathrm{~nm}$ has large contributions above $\log (\tau)=-1$, where the top of the flux tube is located, so that the nonlinear propagation of the spectral effects introduced by the flux tube gives rise to very strong asymmetries. On the other hand, the (much weaker) infrared line of Fe I $1564.8 \mathrm{~nm}$ is formed right around the location of the flux tube.

\section{Variation of the NCP along an azimuthal section}

We now calculate synthetic $V$-profiles along an azimuthal section for a specified radial position. The situation is sketched in Fig. 9: for a given heliocentric angle $\theta$ and a radial position $r$, the azimuthal section of the penumbra is traced out by varying the spot angle $\psi$. It turns out that this azimuthal variation $\mathcal{N}(\psi)$ exhibits an interesting behavior (see also Müller 2001; Schlichenmaier et al. 2002), which differs significantly from Fe I $630.25 \mathrm{~nm}$ to Fe I $1564.8 \mathrm{~nm}$.

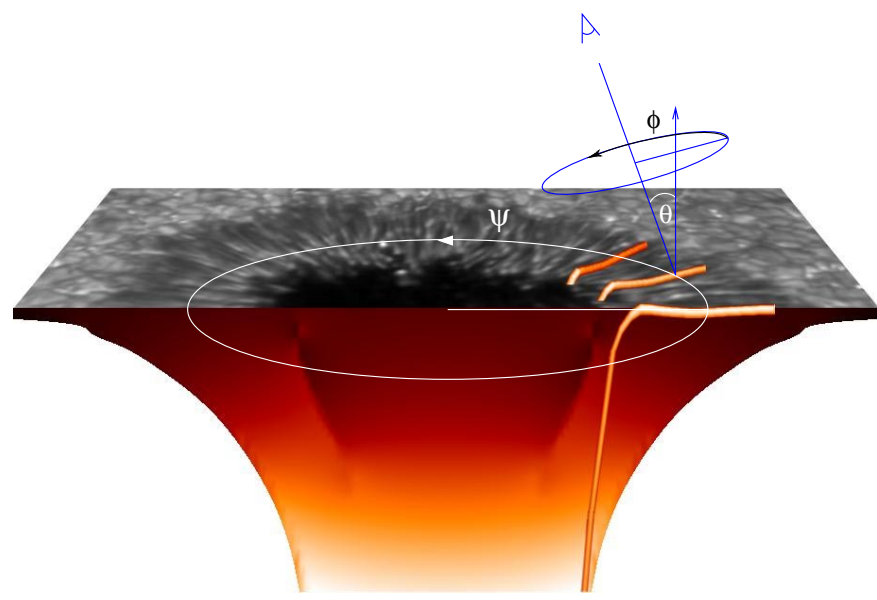

Fig. 9. Magnetic flux tubes in the penumbra. The azimuthal angle $\phi$ and the inclination $\gamma$ of the magnetic field of background and flux tube vary with the spot angle $\psi$. The heliocentric angle is denoted by $\theta$.

For a specified radial position of $12000 \mathrm{~km}$, Fig. 10 displays the results for Fe I $630.25 \mathrm{~nm}$ (left panel) and for Fe I $1564.8 \mathrm{~nm}$ (right panel). The calculations are performed with (solid line) and without (dashed line) the effects of anomalous dispersion for $\theta=15^{\circ}$. For both lines, $\mathcal{N}(\psi)$ is symmetric with
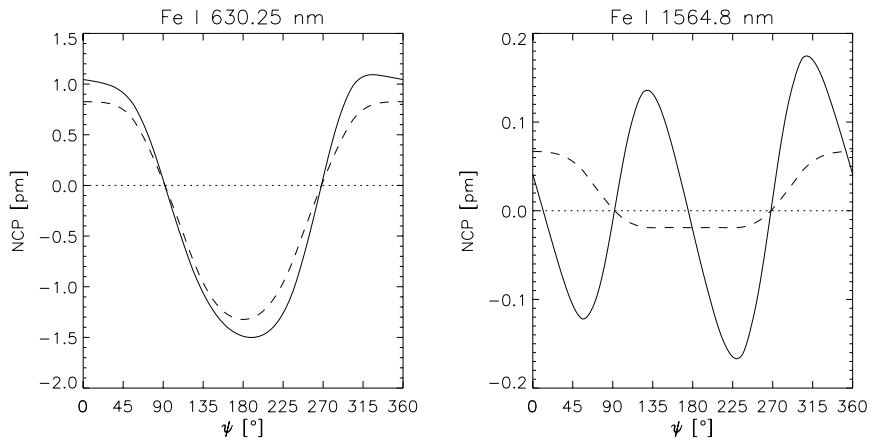

Fig. 10. Left panel: $\mathcal{N}(\psi)$ for Fe I $630.25 \mathrm{~nm}$, with $\theta=15^{\circ}$. Solid line: with anomalous dispersion; dashed line: without anomalous dispersion. Right panel: same as left panel, but for Fe I $1564.8 \mathrm{~nm}$.

respect to the $x$-axis $\left(\psi=0^{\circ}\right.$ and $\left.180^{\circ}\right)$ if the effects of anomalous dispersion are not taken into account. Including anomalous dispersion, this symmetry is broken. For Fe I $630.25 \mathrm{~nm}$, the antisymmetric component is small relative to the symmetric component. For Fe I $1564.8 \mathrm{~nm}$, however, the antisymmetric component dominates $\mathcal{N}(\psi)$.

\subsection{Symmetry properties of $\mathcal{N}(\psi)$}

To understand the antisymmetric component in $\mathcal{N}(\psi)$, we consider the symmetry properties of the discontinuities $\Delta v(\psi)$, $\Delta \gamma(\psi)$, and $\Delta \phi(\psi)$ at the interface between the flux tube and the background, which cause the asymmetry in $V(\lambda)$. For the sake of clarity, we first repeat the most important statements which were made in Schlichenmaier et al. (2002). In our model, $\Delta B$ is negligible at the interface and $B$ only slightly decreases with height. Its influence can therefore be neglected in the following discussion, but we note that $\Delta B(\psi)$ is symmetric and would not alter the following argument.

\subsubsection{Properties of $\Delta \phi(\psi), \Delta \gamma(\psi)$, and $\Delta v(\psi)$}

Taking into account that $\psi=\phi^{\prime}$ for an axially symmetric sunspot with no azimuthal components, it is seen from Eqs. (12), (13), and (14) that $\gamma(\psi)$ and $v(\psi)$ are symmetric, while $\phi(\psi)$ is antisymmetric with respect to the transformation $\psi \rightarrow-\psi$. As a consequence, $\Delta \phi(\psi)=\phi_{\mathrm{t}}(\psi)-\phi_{\mathrm{b}}(\psi)$ is antisymmetric, while $\Delta \gamma(\psi)$ and $\Delta v(\psi)$ are symmetric. The appearance of different signs of $\Delta \phi(\psi)$ within a sunspot is sketched in Fig. 11.

\subsection{2. $\Delta \phi$ is capable of breaking the symmetry}

It has been demonstrated analytically by Landolfi \& Landi Degl'Innocenti (1996) that a discontinuity in the azimuth of the magnetic field vector along the LOS is, in conjunction with a discontinuity in the LOS velocity, capable of producing a nonvanishing net circular polarization, $\mathcal{N}$. Their formulae reflect that a discontinuity in $\phi$ along the LOS can produce a nonzero $\mathcal{N}$, if and only if anomalous dispersion is included in the transfer equation of polarized light. Moreover, they demonstrate that the effect of $\Delta \phi$ on $\mathcal{N}$ is proportional to $\sin (2 \Delta \phi)$, 


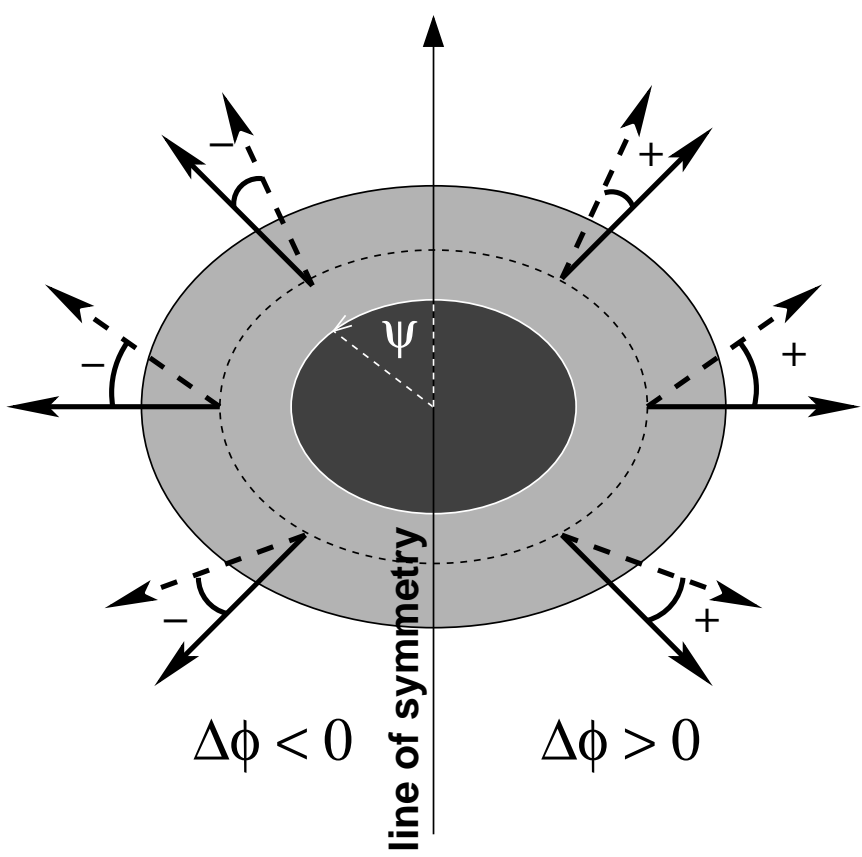

Fig. 11. Pictorial representation of the difference in azimuth, $\Delta \phi(\psi)$, between the magnetic field vector inside a flux tube (solid arrows) and that of the background field (dashed arrows). The tubes are nearly horizontal, while the field vector of the background has a steeper inclination, but is also in the plane spanned by the spot axis and the flux tube axis.

implying that $\mathcal{N}=\mathcal{N}(\Delta \phi)$ is an antisymmetric function. This will be described in more detail in the next section.

Since $\Delta \gamma(\psi)$ and $\Delta v(\psi)$ are both symmetric, $\mathcal{N}=$ $\mathcal{N}(\Delta \gamma(\psi), \Delta v(\psi))$ must also be symmetric with respect to $\psi=0$. Hence, only $\Delta \phi$ is capable of introducing an antisymmetric component in $\mathcal{N}(\psi)$, i.e. $\mathcal{N}(\psi)$ is composed of a symmetric contribution from $\Delta \gamma(\psi)$ and $\Delta v(\psi)$ (and from $\Delta B(\psi)$, if present), and of an antisymmetric contribution from $\Delta \phi(\psi)$. The latter contributes to $\mathcal{N}$ only if anomalous dispersion is included.

It can be seen in Fig. 10 that the values for $\mathcal{N}$ with and without anomalous dispersion are not identical where $\Delta \phi=0$, i.e. for $\psi=0^{\circ}, 180^{\circ}$. This means that $\mathcal{N}$, which is solely produced by $\Delta \gamma$ at these locations, depends on whether anomalous dispersion is included or not. In other words, switching on the anomalous dispersion introduces both a symmetric contribution to $\mathcal{N}(\psi)$ and an antisymmetric contribution that is caused by $\Delta \phi$.

\subsection{Analytical estimation of different contributions to the NCP}

In this section, we introduce the results of the analytical calculations by Landolfi \& Landi Degl'Innocenti (1996) and subsequently apply them to our model. Following their work, we consider a static Milne-Eddington atmosphere on top of which a thin atmospheric layer (slab) is located where plasma flows with a LOS velocity $\omega_{\mathrm{A}}^{(\mathrm{s})}$. Let $\boldsymbol{B}^{(\mathrm{s})} \equiv\left(B_{\mathrm{s}}, \gamma_{\mathrm{s}}, \phi_{\mathrm{s}}\right)$ be the magnetic field of this layer and $\boldsymbol{B} \equiv(B, \gamma, \phi)$ that of the atmosphere below it. The radiative transfer across the slab is described by the equation

$\frac{\mathrm{d} \boldsymbol{I}}{\mathrm{d} \tau}=-\boldsymbol{K}^{(\mathrm{s})} \boldsymbol{I}+\boldsymbol{j}^{(\mathrm{s})}$,

which is formally solved by

$\boldsymbol{I}(0)=\int_{\tau_{\mathrm{s}}}^{0} \boldsymbol{O}(0, \tau) \boldsymbol{j}^{(\mathrm{s})} \mathrm{d} \tau+\boldsymbol{O}\left(0, \tau_{\mathrm{s}}\right) \boldsymbol{I}^{(\mathrm{b})}$

In this expression, $\tau_{\mathrm{s}}$ denotes the optical depth of the layer, $\boldsymbol{O}$ is the evolution operator defined by Eq. (6) and $\boldsymbol{I}^{(\mathrm{b})}$ the Stokes vector illuminating the slab from below. Under the assumption that the flux slab is optically thin $\left(\tau_{\mathrm{s}} \ll 1\right)$, the operator $\boldsymbol{O}$ can be expanded in a power series which yields to first order

$\boldsymbol{O}(0, \tau)=\mathrm{e}^{\tau \boldsymbol{K}^{(\mathrm{s})}} \approx 1+\tau \boldsymbol{K}^{(\mathrm{s})} \quad\left(0 \leq \tau \leq \tau_{\mathrm{s}}\right)$.

For the NCP, one then obtains in lowest order in $\tau_{\mathrm{s}}$

$\mathcal{N}=\tau_{\mathrm{s}} \int\left[\eta_{V}^{(\mathrm{s})} I^{(\mathrm{b})}+\rho_{U}^{(\mathrm{s})} Q^{(\mathrm{b})}-\rho_{Q}^{(\mathrm{s})} U^{(\mathrm{b})}+\eta_{I}^{(\mathrm{s})} V^{(\mathrm{b})}\right] \mathrm{d} \lambda$,

where the index (b) denotes the intensities from the static atmosphere. Under the simplifying assumptions of a MilneEddington atmosphere and a weak spectral line, Landolfi \& Landi Degl'Innocenti derived an analytical expression for a NCP parameter $v=\mathcal{N} / \alpha$, which merely differs from the plain NCP we are using here by a factor of the equivalent width, $\alpha=\int\left[I_{\mathrm{c}}(\tau=0)-I(\tau=0, \lambda)\right] \mathrm{d} \lambda$. Exploiting the properties of convolutions of Voigt- and Faraday-Voigt-functions, three contributions to the NCP can be isolated. They are caused by discontinuities in magnetic field strength, $B$, inclination, $\gamma$ and the azimuth, $\phi$, respectively. Apart from the line absorption coefficient and trigonometric factors, the expressions for the $\Delta B$-effect and the $\Delta \gamma$-effect contain sums of the Voigtfunctions, $H(v, a)$, of the single Zeeman components, while the $\Delta \phi$-effect depends on the corresponding Faraday-Voigtfunctions:

$\Delta B$-effect:

$$
\begin{aligned}
\mathcal{N}_{\Delta B}= & k \cdot \cos \gamma\left\{\left(1+\cos ^{2} \gamma\right)\left[H\left(v_{\mathrm{rr}}, \hat{a}\right)-H\left(v_{\mathrm{bb}}, \hat{a}\right)\right]\right. \\
& +\sin ^{2} \gamma\left[H\left(v_{\mathrm{rp}}, \hat{a}\right)+H\left(v_{\mathrm{pr}}, \hat{a}\right)-H\left(v_{\mathrm{bp}}, \hat{a}\right)\right. \\
& \left.\left.-H\left(v_{\mathrm{pb}}, \hat{a}\right)\right]\right\},
\end{aligned}
$$

$\Delta \gamma$-effect:

$$
\begin{aligned}
\mathcal{N}_{\Delta \gamma}= & k \cdot\left\{[ \operatorname { c o s } \gamma _ { \mathrm { s } } \operatorname { s i n } ^ { 2 } \gamma - \operatorname { c o s } \gamma \operatorname { s i n } ^ { 2 } \gamma _ { \mathrm { s } } ] \left[H\left(v_{\mathrm{rp}}, \hat{a}\right)\right.\right. \\
& \left.-H\left(v_{\mathrm{bp}}, \hat{a}\right)\right]+\frac{1}{2}\left[\cos \gamma_{\mathrm{s}}\left(1+\cos ^{2} \gamma\right)-\cos \gamma\right. \\
& \left.\left.\cdot\left(1+\cos ^{2} \gamma_{\mathrm{s}}\right)\right]\left[H\left(v_{\mathrm{rb}}, \hat{a}\right)-H\left(v_{\mathrm{br}}, \hat{a}\right)\right]\right\}
\end{aligned}
$$

$\Delta \phi$-effect:

$$
\begin{aligned}
\mathcal{N}_{\Delta \phi}= & k \cdot \sin ^{4} \gamma \sin 2\left(\phi-\phi_{s}\right) \cdot\left[\frac{3}{2} L\left(v_{\mathrm{pp}}, \hat{a}\right)-L\left(v_{\mathrm{rp}}, \hat{a}\right)\right. \\
& \left.-L\left(v_{\mathrm{bp}}, \hat{a}\right)+\frac{1}{4} L\left(v_{\mathrm{rb}}, \hat{a}\right)+\frac{1}{4} L\left(v_{\mathrm{br}}, \hat{a}\right)\right] .
\end{aligned}
$$


In these formulae, $k=\alpha \frac{\kappa_{\mathrm{L}}^{(\mathrm{s})} \tau_{\mathrm{s}}}{4 \sqrt{2 \pi}}$ (where $\kappa_{L}$ is the ratio between line and continuum absorption coefficient), $\hat{a}=\sqrt{2} a$ is the damping term, $L(v, a)=2 F(v, a)$ with the Faraday-Voigtfunction, $F(v, a)$, and $v_{i j}$ representing the relative wavelength shifts due to Doppler and Zeeman effect (cf. Landolfi \& Landi Degl'Innocenti 1996). The index p indicates an unshifted component, $r$ and $b$ stand for red- and blue-shifted components, respectively. As we noted in Sect. 5.1, the ratios of Doppler shift and Zeeman splitting are very different for the spectral lines of Fe I $1564.8 \mathrm{~nm}$ and Fe I $630.25 \mathrm{~nm}$ as the first effect is linear in wavelength while the second one is quadratic. These ratios enter the Voigt- and Faraday-Voigt-functions by means of the $v_{i j}$ and give rise to the fact that the asymmetries caused by discontinuities in $B, \gamma$ and $\phi$ have very different weights depending on the wavelength.

This shall be illustrated with typical values of the midpenumbra: For a heliocentric angle of $\theta=10^{\circ}$, magnetic field strengths of $B_{\mathrm{b}}=1700 \mathrm{G}, B_{\mathrm{t}}=1600 \mathrm{G}$ and inclination angles of $\gamma_{\mathrm{b}}=60^{\circ}, \gamma_{\mathrm{t}}=90^{\circ}$ (b: background, t: flux tube), we obtain for Fe I $630.25 \mathrm{~nm}$ :

$\mathcal{N}_{\Delta B}=0.04 \cdot k_{1}$

$\mathcal{N}_{\Delta \gamma}=0.22 \cdot k_{1}$

$\mathcal{N}_{\Delta \phi}=-0.14 \cdot k_{1}$,

while the same calculation yields for Fe I $1564.8 \mathrm{~nm}$ :

$\mathcal{N}_{\Delta B}=1.4 \times 10^{-5} \cdot k_{2}$,

$\mathcal{N}_{\Delta \gamma}=5.2 \times 10^{-6} \cdot k_{2}$,

$\mathcal{N}_{\Delta \phi}=6.0 \times 10^{-1} \cdot k_{2}$.

For the penumbral model used here and averaged line absorption coefficients, $k_{1} / k_{2} \approx 1-2$.

We conclude that the $\Delta \phi$-effect is by far the dominating source of asymmetry for the infrared line, while for Fe I $630.25 \mathrm{~nm}$, the $\Delta \phi$ - and $\Delta \gamma$-effects are of the same strength.

In this context, it has to be noted that the approximation of a weak spectral line is strictly speaking only valid for Fe I $1564.8 \mathrm{~nm}$, and saturation effects come into play for Fe I $630.25 \mathrm{~nm}$. Moreover, discontinuities occur mostly in more than one parameter, so that a complex interplay between different factors results. Despite these simplifications, however, the azimuthal variations of the NCP which we calculate for a penumbral model with flux tube, can be explained and understood on the basis of these analytical considerations.

\section{Synthetic NCP maps and comparison with observations}

In order to gain insight in the spatial distribution of net circular polarization in sunspot penumbrae and to compare our findings with observational results, we use our penumbral model to compute synthetic maps of the NCP. For three different heliocentric angles $\left(\theta=10^{\circ}, 30^{\circ}, 45^{\circ}\right)$, the results are displayed in in the upper two rows of Fig. 12.

\subsection{Computation of synthetic NCP maps}

In the calculations presented here, we have only used the configuration of one flux tube, at a time of evolution when it had reached a nearly stationary state. These maps show the NCP of a flux tube in the penumbra in azimuthal steps of $\Delta \psi=2^{\circ}$ and for 28 radial positions between $r=9500 \mathrm{~km}$ and $r=16000 \mathrm{~km}$. A more refined model would have to account for the structure in azimuthal direction, i.e. the alternation between flux tube and inter-flux-tube space, which is not done here. This simplification does not affect the symmetry consideration but influences the polarization amplitude. The umbra is not part of the model investigated here, and its NCP is set to zero in Fig. 12. For a qualitative comparison with observations, the NCP maps were smoothed using a moving average, so that the spatial resolution corresponds to $1^{\prime \prime}$ which is approximately the pixel-limited resolution of the Tenerife Infrared Polarimeter (ca. 0.8").

The qualitatively different behavior of the two spectral lines is easily recognized: While the NCP of the spectral line Fe I $630.25 \mathrm{~nm}$ is predominantly symmetric with respect to the line connecting disk center and spot center, the NCP of Fe I $1564.8 \mathrm{~nm}$ exhibits a predominantly antisymmetric variation as a function of the spot angle, $\psi$. Moreover, it can be seen that the NCP decreases in the outward direction as a consequence of the decreasing difference in inclination, $\Delta \gamma$. The thin concentric arcs around $r=10000 \mathrm{~km}$ on the center-side, showing a slightly positive NCP $(0<\mathcal{N}<0.04 \mathrm{pm})$ are located at the footpoint of the simulated flux tube which reaches the photosphere at this radial distance. There, the hot upflow gives rise to strong gradients in the temperature and the magnetic field. This effect deserves closer investigation, which will be done in a forthcoming paper. For example, in the vicinity of the footpoint, where the upflowing plasma is still hotter than the background atmosphere but already transparent (i.e. $\log \left(\tau_{c, 500 \mathrm{~nm}}\right)=1$ is reached below the flux tube), spectral lines can be in emission and the Stokes profiles show an apparent inverse polarity. As described in Sect. 8.3, the amplitude of the NCP of Fe I $630.25 \mathrm{~nm}$ is larger than the one of Fe I $1564.8 \mathrm{~nm}$ for all three heliocentric angles shown in Fig. 12, and the modulus of the NCP of Fe I $630.25 \mathrm{~nm}$ is larger on the limb side of the sunspot than on the center side.

\subsection{Comparison with observations}

Maps of the net circular polarization of sunspot penumbrae have been published by Westendorp Plaza et al. (2001) in Fe I $630.25 \mathrm{~nm}$ and by Schlichenmaier \& Collados (2002) in Fe I $1564.8 \mathrm{~nm}$. As an example, one map for each line is displayed in the bottom row of Fig. 12. These measurements reveal that in penumbrae, $\mathcal{N}(\psi)$ is essentially symmetric for Fe I $630.25 \mathrm{~nm}$ and antisymmetric for Fe I $1564.8 \mathrm{~nm}$. As displayed in Fig. 10, the effect of anomalous dispersion also introduces an asymmetry in $\mathcal{N}(\psi)$. We do not intend to make any quantitative comparisons here, especially not concerning the exact amplitude of the NCP. Qualitatively, however, our theoretical results, based on synthetic lines that emanate from the 
(a) Fel $1564.8 \mathrm{~nm}$
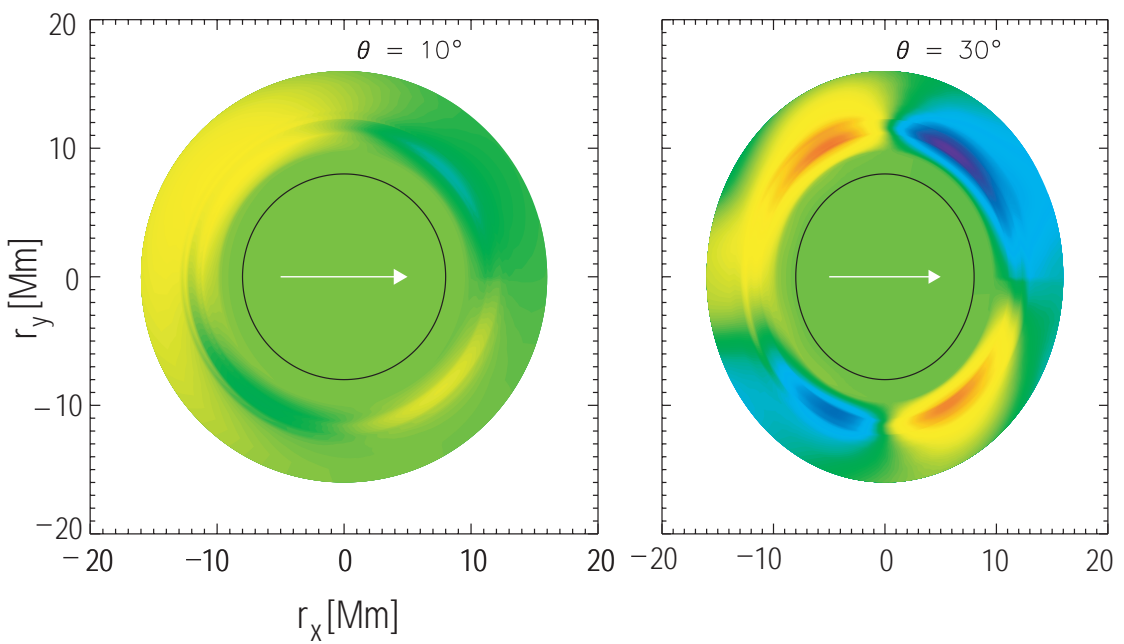

(b) Fe I $630.25 \mathrm{~nm}$

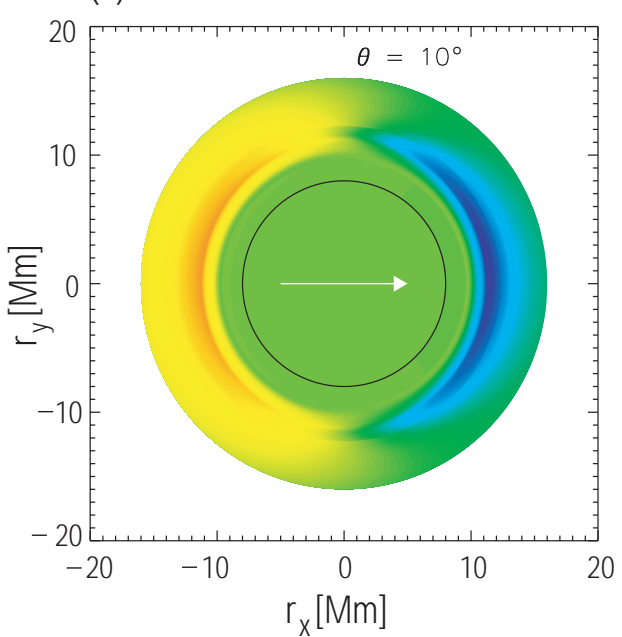

(c) Fe I $1564.8 \mathrm{~nm}$

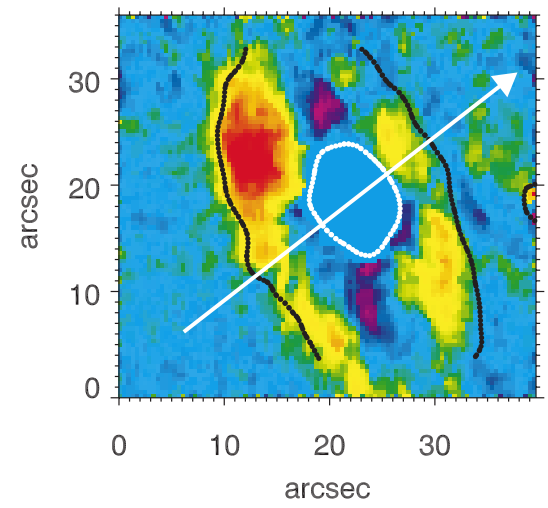

NCP [pm]

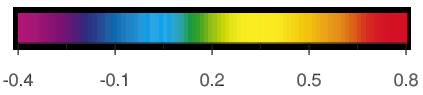

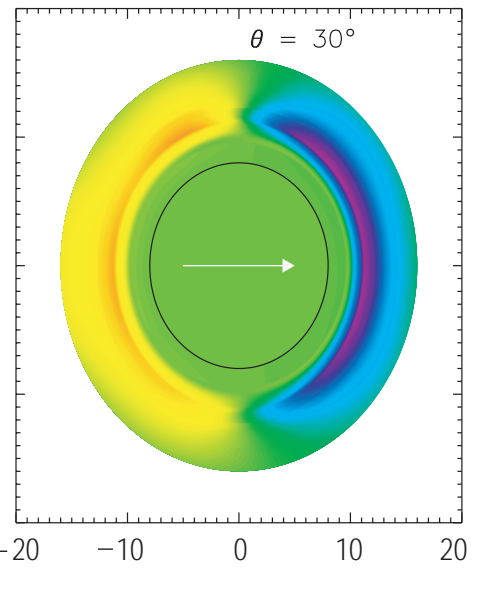

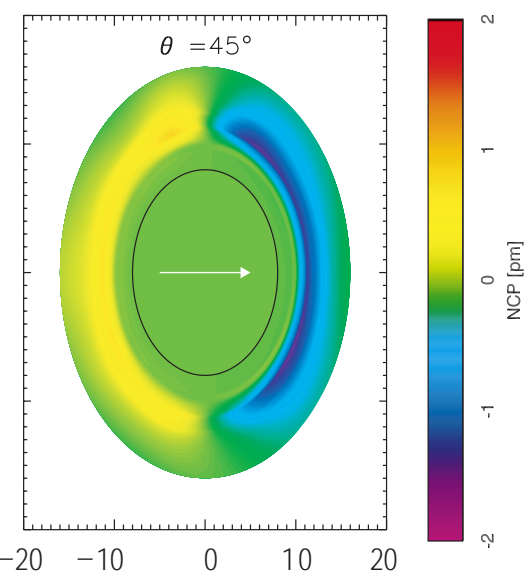

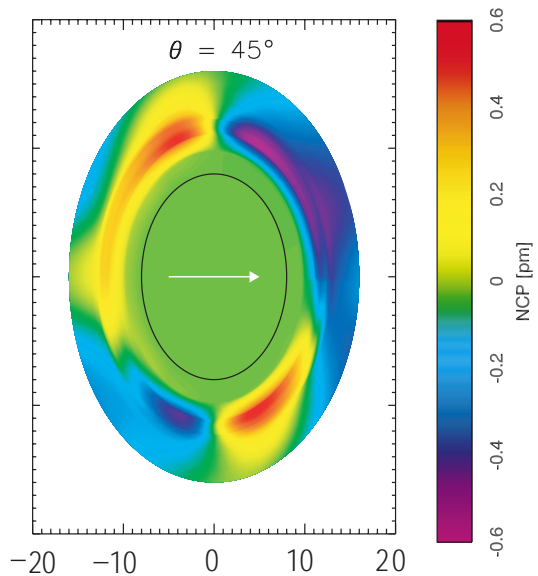

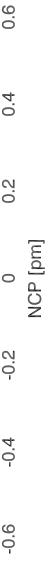

\section{$\frac{\bar{c}}{\frac{E}{0}}$}


moving tube model, are in good agreement with these observations and reproduce the fundamental symmetry properties.

\subsection{Center-to-limb variation of the NCP}

Measurements of the center-to-limb-variation of the NCP have been presented for Fe I $630.25 \mathrm{~nm}$ by, e.g., Martínez Pillet (2000), while in the infrared spectral range, no such measurements have been carried out to date.

We present here calculations of the CLV for both Fe I $630.25 \mathrm{~nm}$ and Fe I $1564.8 \mathrm{~nm}$. The former are qualitatively in good agreement with the observations of Martínez Pillet (2000), while the latter predict a significantly different behavior which awaits a comparison with observations. For such measurements, it is important to be aware that the variation of the NCP along an azimuthal section in the penumbra (as described in Sect. 7) may exceed the CLV for a fixed position, so that the exact azimuthal position in the penumbra for which the CLV is measured must be known. Fig. 13 shows the CLV of the NCP for a radial position of $r=12000 \mathrm{~km}$ and three different azimuthal angles, $\psi$, with respect to the line connecting spot center and disk center. The distinction between limb and center side is accounted for by the definition of the azimuthal angle: $\psi \in\left(-90^{\circ},+90^{\circ}\right)$ denotes a position on the center side, $\psi \in\left(+90^{\circ},+270^{\circ}\right)$ a position on the limb side.

For both spectral lines, it is clearly seen that the CLV curves for center and limb side are not symmetric with respect to each other. This is due to the fact that the projection of the background magnetic field onto the LOS differs in the sense that on the limb side, a sign reversal of the background field occurs, while this is not the case on the center side.

\subsubsection{CLV along the line-of-symmetry}

Let us first concentrate on the upper panel of Fig. 13, which describes the CLV for a point on the line-of-symmetry. The shape of the CLV curves is qualitatively different for Fe I $630.25 \mathrm{~nm}$ and Fe I $1564.8 \mathrm{~nm}: \mathcal{N}_{1}=\mathcal{N}(\mathrm{Fe}$ I $630.25 \mathrm{~nm})$ reaches its extrema on both sides of the penumbra already for small heliocentric angles $\left(\theta=15^{\circ}\right.$ on the center side, $\theta=23^{\circ}$ on the limb side) and decreases monotonically for larger angles. In contrast to that, $\mathcal{N}_{2}=\mathcal{N}(\mathrm{Fe}$ I $1564.8 \mathrm{~nm})$ has a much smaller modulus close to disk center and reaches its extremum on the limb side only for heliocentric angles close to $\theta=90^{\circ}$. On the center side, $\mathcal{N}_{2}$ is maximal for $\mu=0.55\left(\theta=66.6^{\circ}\right)$ and even changes sign for large $\theta$.

The two most important line parameters which cause this different behavior are the excitation potential, $\chi$, and the wavelength, where $\chi(\mathrm{Fe} I 630.25 \mathrm{~nm})=3.69 \mathrm{eV}$ and $\chi(\mathrm{Fe}$ I $1564.8 \mathrm{~nm})=5.43 \mathrm{eV}$. The excitation potential enters the line absorption coefficient, $\kappa_{l}$, as $\kappa_{l} \propto \exp (-\chi / k T)$. For large $\kappa_{l}$, as in the case of Fe I $630.25 \mathrm{~nm}$, the line core saturates and the asymmetry of the Stokes- $V$-profile increases. This explains the shape of the CLV curves close to disk center: Even for small $\theta$, saturation effects play a significant role for Fe I $630.25 \mathrm{~nm}$, while the infrared line remains unaffected (for details, the reader is referred to Müller 2001).
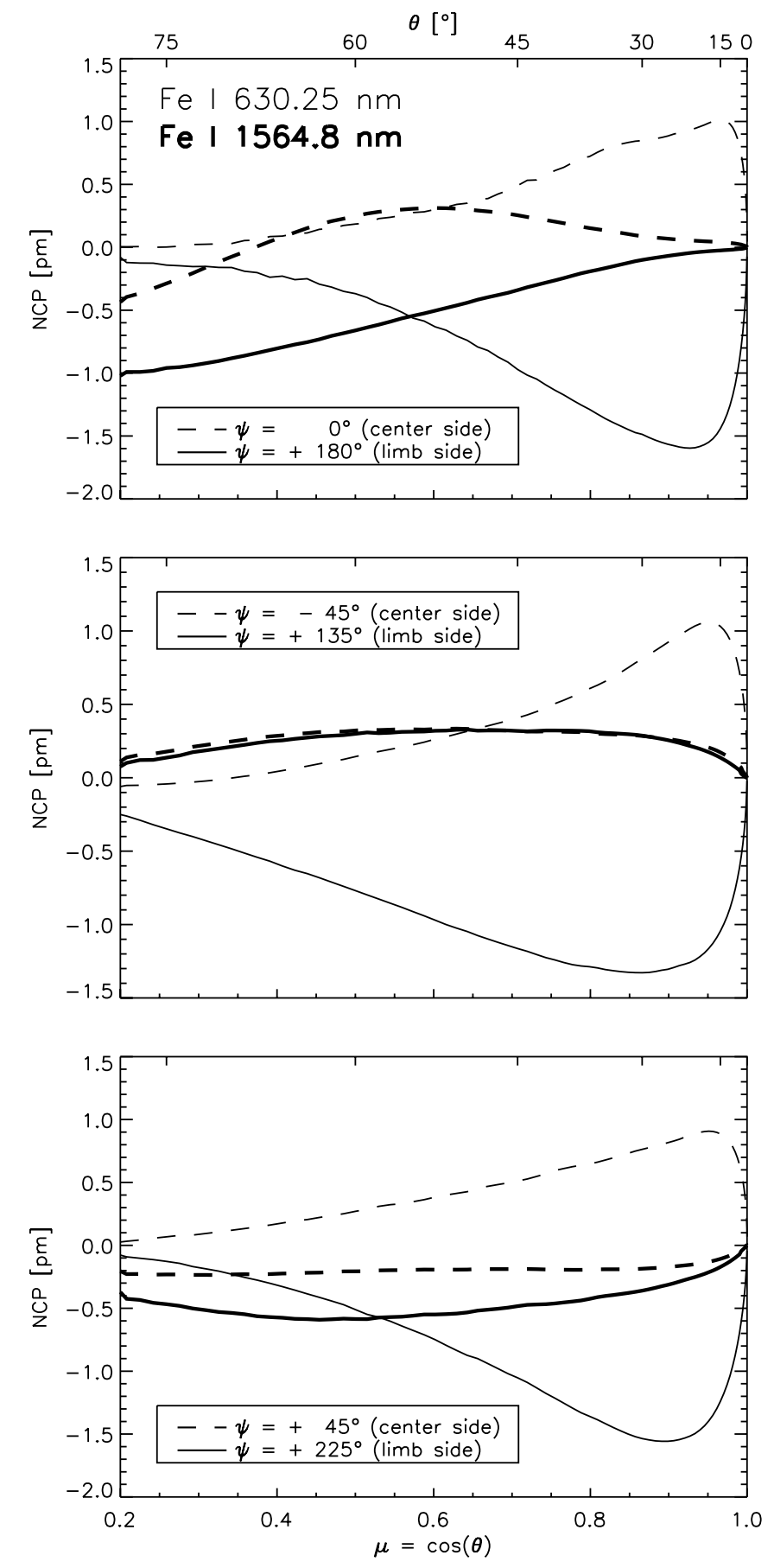

Fig. 13. Center-to-limb variation of the NCP for Fe I $630.25 \mathrm{~nm}$ (thin lines) and Fe I $1564.8 \mathrm{~nm}$ (thick lines) and different azimuthal angles, $\psi$, with respect to the line connecting spot center and disk center.

As described in Sect. 5.1, strongly asymmetric Stokes- $V$ profiles occur if the profile contribution of the flow channel is Doppler-shifted relative to the profile contribution of the background by the same amount as the Zeeman splitting. The heliocentric angle, $\theta$, for which $\Delta \lambda_{\mathrm{D}} / \Delta \lambda_{\mathrm{B}}=1$ is reached, is a function of wavelength $\lambda$. According to Eqs. (22) and (23), this angle amounts to $\theta_{1}=18.4^{\circ}$ for Fe I $630.25 \mathrm{~nm}$ and $\theta_{2}=66.6^{\circ}$ for Fe I $1564.8 \mathrm{~nm}$. This effect is modified due to the fact that the amplitude of the Stokes- $V$ profile varies with the 
heliocentric angle. For the infrared line, this leads to a decrease of $\mathcal{N}(\theta)$ for $\theta>40^{\circ}$ and a sign reversal for large $\theta$. In the case of Fe I $630.25 \mathrm{~nm}$, the same effect shifts the extrema of the CLV from $\theta_{1}=18.4^{\circ}$ to $15^{\circ}$ on the center side and to $23^{\circ}$ on the limb side.

\subsubsection{Zero-crossing of the CLV}

Martínez Pillet (2000) reports that the zero-crossing point of the center-to-limb variation of the NCP of Fe I $630.25 \mathrm{~nm}$ is significantly shifted off disk center $\left(\mu=0.96\right.$, i.e. $\left.\theta \approx 16^{\circ}\right)$ and is located on the center side of the penumbra.

A zero-crossing of the NCP, $\mathcal{N}(\theta)=0$, on the center side of the line-of-symmetry can be reproduced by a static background velocity field and flow channels inclined downwards by an angle of $\gamma^{\prime}=90^{\circ}+\theta_{0}$, with $\theta_{0}$ being the heliocentric angle of the zero-crossing. Then, the LOS component of the velocity vanishes at $\theta_{0}$, and hence, $\mathcal{N}\left(\theta_{0}\right)=0$. If the zero-crossing was on the limb-side, the flow channel would have to be inclined upwards by an angle of $\gamma^{\prime}=90^{\circ}-\theta_{0}$ (for more details, see Müller 2001).

For example, a tube inclination angle of $\gamma^{\prime}=106^{\circ}$ leads to a vanishing NCP on the center-side penumbra at $\theta_{0}=16^{\circ}$ $(\mu=0.96)$. This reproduces the measurements presented in Fig. 2 of Martínez Pillet (2000) (Note that his choice of the sign of the NCP is opposite to ours: In our work, the blue lobe of a umbral Stokes- $V$ profile at disk center is of negative sign.). Thus, assuming the Evershed flow to be confined to flow channels, a zero-crossing of the NCP on the center side of the lineof-symmetry is an indirect evidence of downflows within the penumbra ${ }^{1}$.

Under the assumption that no downflows are present in the penumbra, Martínez Pillet (2000) argues correctly that the observed zero-crossing of the NCP cannot be reproduced by a single flux tube in a static background. If this assumption is dropped, however, this CLV can indeed be reproduced without a velocity field in the background atmosphere. The realizations of the flux tube model which we have investigated here exhibit only horizontal outflows, but recent simulations (Schlichenmaier 2002) show that downflows represent possible solutions as well.

\subsection{CLV for different azimuthal angles}

For azimuthal angles $\psi \neq 0^{\circ}, 180^{\circ}$, the $\Delta \phi$-effect, described in Sect. 7.1, represents an additional source of net circular polarization. The most remarkable feature of the CLV curves which are displayed in the middle and lower panel of Fig. 13 is the fact that for the infrared line, the NCP is of the same sign for both center and limb side. This behavior is the result of the predominantly antisymmetric variation of $\mathcal{N}(\psi)$ and can be verified by comparing Fig. 13 with the synthetic NCP maps in Fig. 12: for a fixed azimuthal angle, center side and limb side refer to regions with NCP of the same sign.

\footnotetext{
1 If, on the other hand, this zero-crossing was present on the limb side, it would be an indication of upflows, but this behavior is not observed.
}

In contrast, for Fe I $630.25 \mathrm{~nm}$, the $\Delta \gamma$-effect is the strongest source of asymmetry and leads to a predominantly symmetric variation of $\mathcal{N}(\psi)$ (cf. Sect. 7.2). This explains the similar shape of the CLV curves for this line for all azimuthal angles, i.e. maximal NCP for small heliocentric angles, and a monotonic decrease for large $\theta$.

We conclude that in the analysis of observed center-to-limb variations of the NCP in sunspot penumbrae, attention must be paid to the azimuthal variation of the NCP, especially in cases of spatial averaging.

\section{Summary}

We have presented a three-dimensional model of sunspot penumbrae with an axisymmetric background field. It proves useful in understanding the spectral signature of magnetic flux tubes in the penumbra. We show that the simultaneous analysis of two spectral lines with significantly different wavelengths helps to disentangle the various effects that give rise to asymmetric Stokes- $V$ profiles. This is due to the different wavelength dependence of Doppler and Zeeman effect. We focus on two atomic spectral lines of neutral iron, Fe I $630.25 \mathrm{~nm}$ and Fe I $1564.8 \mathrm{~nm}$, for which we compute the center-to-limb variation of the NCP. In the case of Fe I $630.25 \mathrm{~nm}$, our findings are in good agreement with observations, while the results for the infrared line Fe I $1564.8 \mathrm{~nm}$ predict a significantly different behavior.

By calculating the variation of the NCP along azimuthal sections of a sunspot penumbra, we demonstrate that a discontinuity in the azimuth, $\Delta \phi$, of the magnetic field vector along the line-of-sight together with the effects of anomalous dispersion plays a crucial role for the interpretation of spectropolarimetric measurements in sunspot penumbrae.

In an axially symmetric sunspot in which the magnetic and velocity field vectors have no azimuthal components, a nearly horizontal flow channel embedded in an inclined magnetic background field introduces a discontinuity, $\Delta \phi$, in the azimuth relative to the line-of-sight. Along an azimuthal section within the penumbra, $\Delta \phi(\psi)$ is antisymmetric with respect to the line-of-symmetry that connects the disk center with the spot center, giving rise to an antisymmetric contribution to the net circular polarization, $\mathcal{N}(\psi)$. $\mathcal{N}(\psi)$ consists of a symmetric contribution from $\Delta \gamma$ (and $\Delta B$ which, however, is negligible in our model configuration) and an antisymmetric contribution from $\Delta \phi$. The wavelength shift ratio between the Doppler and the Zeeman effect determines the relative weights of these two contributions to $\mathcal{N}$. The difference between the symmetry properties of $\mathcal{N}$-maps in Fe I $1564.8 \mathrm{~nm}$ and Fe I $630.25 \mathrm{~nm}$ can therefore be attributed to the large wavelength difference between the two lines.

The striking difference between observed $\mathcal{N}$-maps for the Fe I $1564.8 \mathrm{~nm}$ and the Fe I $630.25 \mathrm{~nm}$ line can be reproduced by synthetic lines that emanate from a model atmosphere which is based on the moving tube model of Schlichenmaier et al. (1998b). In this respect, the present work provides strong evidence that magnetic fields with (at least) two different inclinations with different flow velocities are present in the penumbra. It also demonstrates that the spatial distribution of $\mathcal{N}(r, \psi)$ 
within the penumbra is a valuable diagnostic tool in order to test penumbral models. With this three-dimensional model at hand, we can compute synthetic maps of the NCP in sunspot penumbrae and compare these with observational data. It also opens up the possibility of incorporating a geometrical sunspot model into a spectral inversion code, which would take advantage of the additional information provided by the spatial dependence of the Stokes profiles.

Studying the center-to-limb variation of the $\mathrm{NCP}, \mathcal{N}(\theta)$, we find that the shape of these curves depends both on the ratio of Doppler shift and Zeeman splitting of the particular spectral line, as well as on its excitation potential. The location of the zero-crossing point of $\mathcal{N}(\theta)$ on the line-of-symmetry of a sunspot penumbra represents a diagnostic tool to determine the inclination angle of the Evershed flow: A vanishing NCP on the center-side penumbra, as observed by Martínez Pillet (2000), is an indirect evidence of downflows in the penumbra.

Acknowledgements. We thank U. Grossmann-Doerth for passing on the radiative transfer code DIAMAG and V. Martínez Pillet for providing data from the Advanced Stokes Polarimeter and for helpful comments. R.S. acknowledges a grant by the Deutsche Forschungsgemeinschaft, DFG (LU 706/1-1).

\section{References}

Auer, L. H., \& Heasley, J. N. 1978, A\&A, 64, 67

Beckers, J. M., \& Schröter, E. H. 1969, Sol. Phys., 10, 384

Delaunay, B. 1934, Izvestia Akademia Nauk SSSR, VII Seria, Otdelenie Matematicheskii i Estestvennyka Nauk, 7, 793

Evershed, J. 1909, MNRAS, 69, 454

Gantmacher, F. R. 1959, The Theory of Matrices, vol. 2 (New York)

Grigorjev, V. M., \& Katz, J. M. 1972, Sol. Phys., 22, 119

Grossmann-Doerth, U. 1994, A\&A, 285, 1012

Illing, R. M. E., Landman, D. A., \& Mickey, D. L. 1974, A\&A, 35, 327

Jahn, K., \& Schmidt, H. U. 1994, A\&A, 290, 295
Kemp, J. C. 1970, ApJ, 162, 169

Kjeldseth Moe, O. 1968, in Structure and Development of Solar Active Regions, IAU Symp., 35, 202

Kröner, D. 1997, Numerical Schemes for Conservation Laws (Wiley \& Teubner, Chichester/Stuttgart)

Landi Degl'Innocenti, E. 1976, A\&AS, 25, 379

Landi Degl'Innocenti, E. 2002, in Astrophysical Spectropolarimetry, ed. J. Trujillo-Bueno, F. Moreno-Insertis, \& F. Sánchez (University Press, Cambridge), 1

Landi Degl'Innocenti, E., \& Landi Degl'Innocenti, M. 1981, Il Nuovo Cimento B, 62, 1

Landolfi, M., \& Landi Degl'Innocenti, E. 1996, Sol. Phys., 164, 191

Magain, P. 1986, A\&A, 163, 135

Makita, M. 1979, PASJ, 31, 575

Maltby, P. 1964, Astrophys. Norv., 8, 205

Martínez Pillet, V. 2000, A\&A, 361, 734

Müller, D. A. N. 2001, Diploma Thesis, University of Freiburg

Rees, D. E., Durrant, C. J., \& Murphy, G. A. 1989, ApJ, 339, 1093

Rimmele, T. R. 1995, A\&A, 298, 260

Sánchez Almeida, J., Landi Degl'Innocenti, E., Martínez Pillet, V., \& Lites, B. W. 1996, ApJ, 466, 537

Sánchez Almeida, J., \& Lites, B. W. 1992, ApJ, 398, 359

Schlichenmaier, R. 2002, AN, 323, 303

Schlichenmaier, R., \& Collados, M. 2002, A\&A, 381, 668

Schlichenmaier, R., Jahn, K., \& Schmidt, H. U. 1998a, ApJ, 493, L121

Schlichenmaier, R., Jahn, K., \& Schmidt, H. U. 1998b, A\&A, 337, 897

Schlichenmaier, R., Müller, D. A. N., Steiner, O., \& Stix, M. 2002, A\&A, 381, L77

Schlichenmaier, R., \& Schmidt, W. 2000, A\&A, 358, 1122

Shurcliff, W. A. 1962, Polarized Light: Production and Use (Harvard Univ. Press, Cambridge, Mass.)

Solanki, S. K., \& Montavon, C. A. P. 1993, A\&A, 275, 283

St. John, C. E. 1913, ApJ, 37, 322

Steiner, O. 2000, Sol. Phys., 196, 245

Title, A. M., Frank, Z. A., Shine, R. A., et al. 1993, ApJ, 403, 780

Westendorp Plaza, C., del Toro Iniesta, J. C., Ruiz Cobo, B., et al. 2001, ApJ, 547, 1130

Wittmann, A. 1974, Sol. Phys., 35, 11 